\title{
IDENTITIES IN THE THEORY OF CONFORMAL MAPPING
}

\author{
BY

\section{P. R. GARABEDIAN AND M. SCHIFFER(1)}

Introduction. In the theory of conformal mapping numerous canonical domains are considered upon which a given domain may be mapped. The functions performing this map are functions of the domain considered and might be called domain functions. Numerous relations between domain functions of different types are known; very many of these functions may be constructed from a few fundamental ones, such as Green's and Neumann's functions of the domain and the harmonic measures of the boundary continua. But these fundamental functions themselves are also closely interrelated and permit numerous identities. It is of interest to organize the system of relations between the domain functions into a simple form. This is convenient for the theory of variation of domain functions with their domain; in fact, we obtain often in extremum problems relative to domain functions several different characterizations of the extremum domain, depending on the type of variation applied in the investigation. It is, therefore, essential to be able to reduce one type of equation to another by means of the various identities for domain functions.

An understanding of all identities between domain functions may be obtained by sustained application of Schottky's theory of multiply-connected domains $[15]\left({ }^{2}\right)$. Schottky proved that there is a close relation between the mapping theory of these domains and the theory of closed Riemann surfaces; the identities among domain functions have their complete analogue in the theory of Abelian integrals and might be proved by means of the latter.

It seems, however, that a theory will be of interest which operates only with concepts of conformal mapping and the geometric properties of the functions considered. The functions which prove in such a theory to be the more basic domain functions may be expected to have importance in the general study of conformal mapping, too. In fact, it will be seen that one of the most fundamental functions in the theory will be a kernel function. This type of function has been studied from various points of view recently $[1,2$, 13]. The development in this paper gives further a new understanding of variation formulas which have been applied frequently in conformal mapping; it allows us also to carry out variational methods in extremum problems with additional conditions, such as the invariance of conformal type.

Presented to the Society, April 17, 1948; received by the editors January 8, 1948.

(1) Paper done under contract with the Office of Naval Research.

(2) Numbers in brackets refer to the bibliography at the end of the paper. 
It is difficult to say how much of the methods for obtaining identities applied in this paper is new. Several authors considered similar domain functions and investigated them by analogous methods; in particular, Grunsky used in his papers combinations of domain functions which are closely related to those applied here $[6,7]$. However, we felt for ourselves the need to carry out a systematic study of these formal identities and to establish a more or less unified method. The identities derived in this paper are far from complete, but we hope that the methods for obtaining them have been worked out clearly enough to facilitate the establishment of further identities which might be required.

1. The fundamental domain functions. Let $D$ be a domain in the complex z-plane bounded by $n$ proper continua $C_{\nu}(\nu=1,2, \cdots, n)$ which form together the boundary $C=\sum_{\nu=1}^{n} C_{\nu}$ of $D$. For the sake of simplicity and without any loss of generality, we shall assume that $D$ is bounded and that each $C_{\nu}$ is a smooth closed curve. We define two univalent functions $\Phi(z ; u, v)$ and $\Psi(z ; u, v)$ by the following requirements: (a) $\Phi(z ; u, v)$ maps $D$ upon the entire complex plane slit along concentric circular arcs around the origin so that the point $u \in D$ corresponds to the origin and the point $v \in D$ to infinity. The residue of the simple pole at $v$ is 1 . (b) $\Psi(z ; u, v)$ maps $D$ upon the entire complex plane slit along rectilinear segments directed towards the origin so that the point $u \in D$ corresponds to the origin and the point $v \in D$ to infinity. The residue of the simple pole at $v$ is 1 .

Existence and uniqueness of these two domain functions is well known. Let us consider the logarithms of both functions of $z \in D$. We have

$$
\log \Phi(z ; u, v)=\log \frac{z-u}{z-v}+F(z ; u, v),
$$

where $F(z ; u, v)$ is regular and single-valued in $D$, and because of the condition on the residue at $z=v$, we have

$$
\log (v-u)+F(v ; u, v)=0 .
$$

If the point $z$ lies in the boundary continuum $C_{\nu}$, we have by definition of $\Phi(z ; u, v)$

$$
\log \Phi(z ; u, v)=\kappa_{\nu}(u, v)+i r_{\nu}(z ; u, v), \quad u, v \in D, z \in C_{p},
$$

where $\kappa_{\nu}$ and $r_{\nu}$ are both real-valued nonanalytic functions of their arguments.

In the same way, we have for $\Psi(z ; u, v)$ the equations

$$
\log \Psi(z ; u, v)=\log \frac{z-u}{z-v}+G(z ; u, v),
$$

with regular and single-valued $G(z ; u, v)$ for $z \in D$, and

$$
\log (v-u)+G(v ; u, v)=0 .
$$


For $z \in C_{\nu}$, we have in this case

$$
\log \Psi(z ; u, v)=s_{\nu}(z ; u, v)+i \lambda_{\nu}(u, v), \quad u, v \in D, z \in C_{v} .
$$

Here $s_{\nu}(z ; u, v)$ and $\lambda_{\nu}(u, v)$ are again real-valued functions of their arguments.

Consider the class $\Omega$ of all functions $f(z)$ which are analytic, regular and single-valued in $D$ and for which the integral

$$
\left(f, f^{+}\right)=\iint_{D}\left|f^{\prime}(z)\right|^{2} d x d y, \quad z=x+i y, \quad f^{\prime}(z)=\frac{d}{d z} f(z),
$$

taken in the Lebesgue sense, exists and is finite $\left({ }^{3}\right)$. We may introduce a metric into the linear space of all functions of class $\Omega$ by defining the scalar product

$$
\left(f, g^{+}\right)=\iint_{D} f^{\prime}(z)\left(g^{\prime}(z)\right)+d x d y
$$

of two arbitrary elements $f(z)$ and $g(z)$ in $\Omega$. We may easily transform the surface integral (6) into a line integral by means of Green's theorem. We obtain

$$
\left(f, g^{+}\right)=\frac{1}{2 i} \oint_{C} f^{\prime}(z)(g(z))^{+} d z=-\frac{1}{2 i} \oint_{C} f(z)\left(g^{\prime}(z)\right)^{+} d z^{+} .
$$

Of course, not for every pair of functions of class $\Omega$ may this integration by parts really be carried out; it is, however, permissible if $f(z)$ and $g(z)$ are both continuously differentiable in $D+C$. On the other hand, our second formula for the scalar product permits the extension of the metric to functions which are meromorphic in $D$ and such that the representation (7) is defined.

In particular, we consider now the expressions $\left(f,(\log \Phi)^{+}\right)$and $\left(f,(\log \Psi)^{+}\right)$. These are well defined by $(7)$, since $\log \Phi$ and $\log \Psi$ are differentiable and single-valued on $C$. We have

$$
\left(f(z),(\log \Phi(z ; u, v))^{+}\right)=\frac{1}{2 i} \oint_{c} f^{\prime}(z)(\log \Phi(z ; u, v))^{+} d z,
$$

which leads, because of (2) and the single-valuedness of $f(z)$ in $D$, to

$$
\begin{aligned}
\left(f(z),(\log \Phi(z ; u, v))^{+}\right) & =-\frac{1}{2 i} \oint_{C} f^{\prime}(z) \log \Phi(z ; u, v) d z \\
& =\frac{1}{2 i} \oint_{C} f(z) d \log \Phi(z ; u, v) .
\end{aligned}
$$

By virtue of the residue theorem this leads finally to

(3) In this paper the conjugate of a complex term $A$ will be denoted by $A^{+}$. 


$$
\left(f(z),(\log \Phi(z ; u, v))^{+}\right)=\pi[f(u)-f(v)] .
$$

Analogously, we have

$$
\left(f(z),(\log \Psi(z ; u, v))^{+}\right)=\frac{1}{2 i} \oint_{c} f^{\prime}(z)(\log \Psi(z ; u, v))^{+} d z,
$$

and, in view of (4),

$$
\begin{aligned}
\left(f(z),(\log \Psi(z ; u, v))^{+}\right) & =\frac{1}{2 i} \oint_{c} f^{\prime}(z) \log \Psi(z ; u, v) d z \\
& =-\frac{1}{2 i} \oint_{c} f(z) d \log \Psi(z ; u, v)
\end{aligned}
$$

and finally

$$
\left(f(z),(\log \Psi(z ; u, v))^{+}\right)=-\pi[f(u)-f(v)] .
$$

From formulas (9) and (11) two interesting combinations of $\log \Phi$ and $\log \Psi$ present themselves because of their simple properties:

$$
\begin{aligned}
& P(z ; u, v)=2^{-1}[\log \Phi(z ; u, v)-\log \Psi(z ; u, v)], \\
& Q(z ; u, v)=2^{-1}[\log \Phi(z ; u, v)+\log \Psi(z ; u, v)] .
\end{aligned}
$$

The function $P(z ; u, v)$ is regular and single-valued for $z \in D$, since the logarithmic poles at $u$ and $v$ cancel by subtraction. Because of $\left(1^{\prime}\right)$ and $\left(3^{\prime}\right)$ one has further

$$
P(v ; u, v)=2^{-1}[F(v ; u, v)-G(v ; u, v)]=0,
$$

and from (9) and (11) we conclude

$$
\left(f(z),(P(z ; u, v))^{+}\right)=\pi[f(u)-f(v)] .
$$

The identity (14) has been derived for functions $f(z) \in \Omega$ which are continously differentiable in $D+C$; but by the usual considerations of approximation it may be easily extended to the whole class $\Omega$. Since $P(z ; u, v)$ belongs itself to the class $\Omega$ we may use in (14) either definition (6) or (7) for the scalar product.

The function $Q(z ; u, v)$ has logarithmic poles at $z=u$ and $z=v$ and is not even single-valued in $D$. Each determination of it is, however, single-valued on $C$; it changes only by an integral multiple of $2 \pi i$ if the point $z$ makes a circuit around $u$ or $v$. From (9) and (11) we conclude

$$
\left(f(z),(Q(z ; u, v))^{+}\right)=0 .
$$

In order to extend this identity to the whole class $\Omega$, we have to replace the line integral occurring in the definition (7) of the scalar product by an integral over the interior of the domain $D$. For this purpose consider the improper 
integral

$$
J\left(f, Q^{+}\right)=\iint_{D} f^{\prime}(z)\left(Q^{\prime}(z ; u, v)\right)+d x d y, \quad Q^{\prime}(z ; u, v)=\frac{d}{d z} Q(z ; u, v) .
$$

If $f(z)$ is continuously differentiable over $D+C$ we may evaluate this integral by integration by parts. We obtain easily

$$
\begin{aligned}
J\left(f, Q^{+}\right) & =-\frac{1}{2 i} \oint_{c} f(z)\left(Q^{\prime}(z ; u, v)\right)^{+} d z^{+}+\pi[f(v)-f(u)] \\
& =\left(f, Q^{+}\right)+\pi[f(v)-f(u)] .
\end{aligned}
$$

This formula permits us to define the scalar product $\left(f, Q^{+}\right)$by means of a surface integral which has a meaning for every function $f(z) \in \Omega$. It is now possible to extend the identity (15) to the whole class $\Omega$.

The most important relation between the two functions $P(z ; u, v)$ and $Q(z ; u, v)$ results from the equations (2) and (4). We obtain from them easily the equation

$$
\begin{gathered}
P(z ; u, v)=-(Q(z ; u, v))^{+}+\left(k_{\nu}(u, v)\right)^{+}, \\
k_{\nu}(u, v)=\kappa_{\nu}(u, v)+i \lambda_{\nu}(u, v),
\end{gathered}
$$

if $\boldsymbol{z}$ lies on the boundary continuum $C_{\nu}$ and $u, v$ are arbitrary points in $D$. We shall make use of this relation in a systematic way and we shall derive from it numerous identities for the functions $P, Q$, and other closely related functions. It is this formula which makes the pair of domain functions $P$ and $Q$ the most convenient basis for an investigation of the fundamental domain functions connected with conformal mapping.

We make a first application of (16) in order to determine the norm of $Q(z ; u, v)$ in our metric (7). We find

$$
\left(Q(z ; u, v),(Q(z ; u, v))^{+}\right)=\frac{1}{2 i} \oint_{c}(Q(z ; u, v))^{+} d Q(z ; u, v),
$$

and using (16) and remarking that

$$
\oint_{c,} d Q(z ; u, v)=0
$$

we obtain

$$
\begin{aligned}
\left(Q(z ; u, v),(Q(z ; u, v))^{+}\right) & =\frac{1}{2 i} \oint_{c} P(z ; u, v) d(P(z ; u, v))^{+} \\
& =-\left(P(z ; u, v),(P(z ; u, v))^{+}\right) .
\end{aligned}
$$

Finally, in view of (14) and $\left(12^{\prime}\right)$, we arrive at 


$$
\left(Q(z ; u, v),(Q(z ; u, v))^{+}\right)=-\left(P(z ; u, v),(P(z ; u, v))^{+}\right)=-\pi P(u ; u, v) .
$$

Since the norm of every function $f(z) \in \Omega$ is non-negative by virtue of definition (5), and since $P$ is of this class, we conclude

$$
P(u ; u, v) \geqq 0, \quad\left(Q(z ; u, v),(Q(z ; u, v))^{+}\right) \leqq 0 .
$$

We see that the function $Q(z ; u, v)$ with logarithmic poles at $u$ and $v$ has a nonpositive norm.

Returning to the class $\Omega$ we remark that the only functions $f(z) \in \Omega$ with vanishing norm are the constants. In order to build up a theory of orthonormal systems it is important to reduce the class $\Omega$ to a subclass in which the vanishing of $\left(f, f^{+}\right)$implies $f \equiv 0$. Such a subclass can be defined in different ways. The most obvious one is to distinguish the point $v \in D$ and to define as subclass $\Omega_{v} \subset \Omega$ the class of those functions $f(z) \in \Omega$ which vanish at the point $v$. Because of $\left(12^{\prime}\right)$ the function $P(z ; u, v)$ belongs to this class. Now it is obvious that if $f(z) \in \Omega_{v}$ has the norm zero it is a constant, and since it vanishes at $v$ it must be identically zero. From (14) we deduce further

$$
\left(f(z),(P(z ; u, v))^{+}\right)=\pi f(u), \quad f(z) \in \Omega_{v} .
$$

It is now always possible to determine a complete orthonormal system for the class $\Omega_{v}$; that is, there exists a system $\left\{f_{v}(z)\right\}$ of functions in $\Omega_{v}$ which satisfies the conditions

$$
\left(f_{\nu}(z),\left(f_{\mu}(z)\right)^{+}\right)=\delta_{\nu \mu}, \quad \delta_{\nu \mu}=\left\{\begin{array}{l}
1, \nu=\mu, \\
0, \nu \neq \mu,
\end{array}\right.
$$

and such that every function $f(z) \in \Omega_{v}$ may be developed in a Fourier series

$$
f(z)=\sum_{\nu=1}^{\infty} a_{\nu} f_{\nu}(z), \quad a_{\nu}=\left(f, f_{\nu}^{+}\right),
$$

which converges uniformly in every closed subdomain of $D$.

Since $P(z ; u, v)$ is of the class $\Omega_{v}$, we may apply to it the development (20) and, in view of $\left(14^{\prime}\right)$, we find

$$
P(z ; u, v)=\pi \sum_{\nu=1}^{\infty} f_{\nu}(z)\left(f_{\nu}(u)\right)^{+} .
$$

This shows that the function $P(z ; u, v)$ is the kernel function of every complete orthonormal system with respect to $\Omega_{v}$. It is remarkable that the kernel function is independent of the choice of the particular system and that it coincides with a function defined in terms of canonical mapping functions.

From $(21)$ it follows further that $P(z ; u, v)$ is anti-analytic in $u$. The dependence of $P(z ; u, v)$ on $v$ is much more complicated, since $v$ enters already into the definition of the basic system $\left\{f_{\nu}(z)\right\}$. We shall see later that the class $\Omega$ may be reduced in a way which does not distinguish the point $v$, and 
that the kernel function of the class obtained is anti-analytic in $u$ and $v$. The importance of (21) for an effective computational construction of $P(z ; u, v)$ needs not to be stressed. The formula is still more interesting because it is possible to compute the second fundamental domain function $Q(z ; u, v)$ by simple integration from the function $P(z ; u, v)$.

In fact, let us represent $Q(z ; u, v)$ in the form

$$
Q(z ; u, v)=\log \frac{z-u}{z-v}+Q_{1}(z ; u, v),
$$

where $Q_{1}(z ; u, v)$ is of class $\Omega$. Applying now (15) to the particular function $P(z ; a, b)$ of class $\Omega$ and using the symmetry law $\left(f, g^{+}\right)=\left(g, f^{+}\right)^{+}$, we find

$$
\left(\log \frac{z-u}{z-v},(P(z ; a, b))^{+}\right)+\left(Q_{1}(z ; u, v),(P(z ; a, b))^{+}\right)=0 .
$$

On the other hand, application of (14) to the particular function $Q_{1}(z ; u, v)$ leads to

$$
\left(Q_{1}(z ; u, v),(P(z ; a, b))^{+}\right)=\pi\left[Q_{1}(a ; u, v)-Q_{1}(b ; u, v)\right] .
$$

From (23) and $\left(23^{\prime}\right)$ we derive

$$
Q_{1}(a ; u, v)-Q_{1}(b ; u, v)=-\frac{1}{\pi}\left(\log \frac{z-u}{z-v},(P(z ; a, b))^{+}\right)
$$

and by definition (22) finally

$$
\begin{aligned}
& Q(a ; u, v)-Q(b ; u, v) \\
& \quad=\log \frac{a-u}{a-v}-\log \frac{b-u}{b-v}-\frac{1}{\pi}\left(\log \frac{z-u}{z-v},(P(z ; a, b))^{+}\right) .
\end{aligned}
$$

This formula determines $Q(z ; u, v)$ up to an additive constant. We shall see later that the combination $Q(a ; u, v)-Q(b ; u, v)$ is of great interest for the general theory because of its symmetry properties. We note further that the scalar product in (25) is to be understood in the sense of a contour integral, since $\log ((z-u) /(z-v))$ has singularities in $D$. One recognizes that the function $Q(a ; u, v)-Q(b ; u, v)$ is analytic in all four variables; another proof for this fact will be given later as application of a more general theory.

2. Some extremum problems in conformal mapping. Consider the family $\mathfrak{F}$ of all functions $F(z)$ which are regular in $D$ except for logarithmic poles at the points $z=u$ and $z=v$ and which have the form

$$
F(z)=\log \frac{z-u}{z-v}+F_{1}(z)
$$

where $F_{1}(z)$ is of class $\Omega$. We assume further that 


$$
\log (v-u)+F_{1}(v)=0,
$$

that is, that the function $\exp \{F(z)\}$ has a simple pole at $\alpha=v$ with residue 1 . In view of (22), we may write every function $F(z) \in \mathfrak{F}$ also in the form

$$
F(z)=Q(z ; u, v)+F_{2}(z),
$$

where $F_{2}(z)$ is again of class $\Omega$, and since $\exp \{Q(z ; u, v)\}$ has at $z=v$ the residue 1 , we have

$$
F_{2}(v)=0 .
$$

We may now consider the norms

$$
\begin{aligned}
\left(F, F^{+}\right)= & \left(Q(z ; u, v),(Q(z ; u, v))^{+}\right)+\left(Q(z ; u, v),\left(F_{2}(z)\right)^{+}\right) \\
& +\left(F_{2}(z),(Q(z ; u, v))^{+}\right)+\left(F_{2}, F_{2}^{+}\right),
\end{aligned}
$$

which are well defined for the family $\mathfrak{F}$ because of $\left(15^{\prime \prime}\right)$ and $\left(18^{\prime}\right)$. We have in view of (15) and (18')

$$
\left(F, F^{+}\right)=-\pi P(u ; u, v)+\left(F_{2}, F_{2}^{+}\right) .
$$

Since $F_{2}(z)$ is of class $\Omega_{v}$ and has, therefore, a non-negative norm, we obtain the result:

THEOREM I. For every function $F(z) \in \mathfrak{F}$ we have $\left(F, F^{+}\right) \geqq-\pi P(u ; u, v)$. Equality holds only for $F(z)=Q(z ; u, v)$.

If $F(z)$ is continuously differentiable in $D+C$, we may write

$$
-\left(F, F^{+}\right)=-\frac{1}{2 i} \oint_{C}(F(z))^{+} d F(z),
$$

and this expression has an easy geometric interpretation. The function $\zeta=F(z)$ maps $D$ conformally (but not necessarily univalently) upon a domain $\Delta$, and the curves $C_{\nu}$ are transformed into new sets of boundary curves $\Gamma_{i}$ $(i=1,2, \cdots)$; each set is obtained from a basic set $\Gamma_{1}, \Gamma_{2}, \cdots, \Gamma_{n}$ by simple translation by an integral multiple of $2 \pi i$. The set $\Gamma_{\nu}(\nu=1, \cdots, n)$ encloses a certain part of the $\zeta$-plane and its area is $-\left(F, F^{+}\right)$. The function $\exp \{F(z)\}$ maps $D$ upon a domain bounded by $n$ curves and we may call $-\left(F, F^{+}\right)$the logarithmic area of the continua enclosed by them. Thus, we have the result:

ThEOREM Ia. If $F(z) \in \mathfrak{F}$, the function $\exp \{F(z)\}$ maps $D$ upon a domain whose complement has a logarithmic area not greater than $\pi P(u ; u, v)$. Only the mapping $\exp \{Q(z ; u, v)\}$ yields the maximal value for the logarithmic area of the complement.

We shall prove that the function $\exp \{Q(z ; u, v)\}$ is univalent in $D$. Since the above theorem is a fortiori true for univalent mappings, we obtain the interesting corollary: 
Theorem Ib. If $D$ is mapped univalently so that $z=u$ corresponds to the origin, $z=v$ to infinity, and so that the residue of the pole at $z=v i s 1$, then the logarithmic area of the complement of the image domain is not greater than $\pi P(u ; u, v)$. This upper limit is attained by the univalent function $\exp \{Q(z ; u, v)\}$.

In order to prove the univalency of $\exp \{Q(z ; u, v)\}$, we return to the definition (13) of $Q(z ; u, v)$. If $z$ lies on a fixed boundary continuum $C_{\nu}$ the abscissa of the point $Q(z ; u, v)$ is determined by $\log \Psi(z ; u, v)$ and the ordinate by $\log \Phi(z ; u, v)$ up to a fixed additive constant. By its geometric definition $\log \Psi$ attains every value on $C_{\nu}$ twice and so does $\log \Phi$. This shows easily that $Q(z ; u, v)$ maps $C_{\nu}$ upon a curve without self-intersection which is cut by each parallel to the real or imaginary axis exactly twice. Since the imaginary part of $\log \Phi$ varies on $C_{\nu}$ by less than $2 \pi$, it follows that $\exp \{Q(z ; u, v)\}$ maps each $C_{\nu}$ in a one-to-one manner upon a simple closed curve. It is further clear that the mapping covers the point at infinity and the origin exactly once. If we can prove that $\exp \{Q(z ; u, v)\}$ has nowhere in $D$ a vanishing derivative, it follows from elementary topological considerations that this function is univalent in $D$.

For the last step in our reasoning we remark that the function

(30) $\log S(z ; u, v)=[\cos \alpha \log \Phi(z ; u, v)+i \sin \alpha \log \Psi(z ; u, v)] e^{-i \alpha}, \alpha$ real,

is the logarithm of a univalent function in $D$. In fact, the right-hand side of (30) is a multivalued function with periods $m \cdot 2 \pi i$. The boundary continuum $C_{\nu}$ is mapped by $\log S$ upon a rectilinear segment, which is transformed into a logarithmic spiral slit if we consider the map by $S(z ; u, v)$. This mapping function has been thoroughly investigated $[4,6]$. We use here only the fact that the derivative of $S$ is not zero for any $z \in D$. Hence, we find

$$
J(z ; u, v)=\frac{\Phi^{\prime}(z ; u, v)}{\Phi(z ; u, v)} \cdot \frac{\Psi(z ; u, v)}{\Psi^{\prime}(z ; u, v)} \neq-i \tan \alpha, \quad z \in D, \alpha \text { real. }
$$

This shows that $J(z ; u, v)$ is never imaginary for $z \in D$. For $z=u$ and $z=v$ one has $J=1$. This shows that

$$
\operatorname{Re}\{J(z ; u, v)\}>0, \quad z \in D .
$$

For if there were a point $z \in D$ with negative $\operatorname{Re}\{J\}$ we could connect it by a continuous curve with $z=u$ and there would exist a point on this curve where $\operatorname{Re}\{J\}=0$. But this is a contradiction to (31), which proves finally (32).

In particular, we conclude from (32) that

$$
\frac{d}{d z}[a \log \Phi(z ; u, v)+b \log \Psi(z ; u, v)] \neq 0, \quad z \in D ; a, b>0 .
$$

From our preceding considerations follows therefore easily: 
Theorem II. Every function $\exp \{a \log \Phi(z ; u, v)+b \log \Psi(z ; u, v)\}$ is univalent in $D$, if $a>0, b>0$, and $a+b=1$. In particular, $\exp \{Q(z ; u, v)\}$ is univalent in $D$.

It is now of interest to study the univalent functions of the above type in more detail and to characterize them by extremum properties. We may write in view of (12) and (13)

$$
\begin{aligned}
\log T(z) & =a \log \Phi(z ; u, v)+b \log \Psi(z ; u, v) \\
& =(a-b) P(z ; u, v)+(a+b) Q(z ; u, v) .
\end{aligned}
$$

For $a+b=1$, we obtain in $T(z)$ a univalent function with $(\log T) \in \mathfrak{F}$, since $\exp \{Q(z ; u, v)\}$ has at $z=v$ the residue 1 and $P(v ; u, v)=0$. In the neighborhood of $z=u$, we have in view of (22)

$$
\begin{aligned}
\log T(z)= & \log (z-u)-\log (u-v)+Q_{1}(u ; u, v) \\
& +(a-b) P(u ; u, v)+O(|z-u|) .
\end{aligned}
$$

Since $P(u ; u, v)>0$, we may by appropriate choice of $a$ and $b$ obtain for $\log T(z)$ a development

$$
\log T(z)=\log (z-u)+\gamma+O(|z-u|)
$$

with an arbitrarily prescribed possible value for $\operatorname{Re}\{\gamma\}$. For it is well known [12] that the largest possible value of $\operatorname{Re}\{\gamma\}$ is attained by $\log \Phi(z ; u, v)$ and the smallest by $\log \Psi(z ; u, v)$, corresponding to a choice of $a=1$ or $b=1$, respectively.

Consider now the subclass $\mathfrak{F}_{\gamma}$ of all functions $F(z) \in \mathfrak{F}$ which have at $z=u$ a given value $\operatorname{Re}\{\boldsymbol{\gamma}\}$. They may be written in the form

$$
F(z)=\log T(z)+F_{2}(z),
$$

where $T(z)$ is the uniquely defined combination of $P$ and $Q$ in the family $\mathfrak{F}_{\gamma}$ and $F_{2}(z)$ is of the class $\Omega$. Obviously, we have by definition

$$
F_{2}(v)=0, \quad F_{2}(u)=\text { imaginary. }
$$

Let us compute now the norm of $F(z)$. We have, in view of (34) and (35),

$$
\begin{aligned}
\left(F, F^{+}\right)= & \left(Q+(a-b) P+F_{2},\left(Q+(a-b) P+F_{2}\right)^{+}\right) \\
= & \left(Q, Q^{+}\right)+\left(Q,\left((a-b) P+F_{2}\right)^{+}\right)+\left((a-b) P+F_{2}, Q^{+}\right) \\
& +\left((a-b) P+F_{2},\left((a-b) P+F_{2}\right)^{+}\right) .
\end{aligned}
$$

Applying now $\left(18^{\prime}\right),(15)$ and (14), we obtain

$$
\begin{aligned}
\left(F, F^{+}\right)= & -\pi P(u ; u, v)+(a-b)^{2} \pi P(u ; u, v) \\
& +(a-b) \pi\left(F_{2}(u)+\left(F_{2}(u)\right)^{+}\right)+\left(F_{2}, F_{2}^{+}\right) .
\end{aligned}
$$

Taking account of $\left(35^{\prime}\right)$ and of the equation $a+b=1$, we arrive finally at 


$$
\left(F, F^{+}\right)=-4 \pi a b P(u ; u, v)+\left(F_{2}, F_{2}^{+}\right) .
$$

Since $\left(F_{2}, F_{2}{ }^{+}\right) \geqq 0$ and equality holds only for $F_{2} \equiv 0$, we have

$$
-\left(F, F^{+}\right) \leqq 4 \pi a b P(u ; u, v),
$$

and equality holds only for $F(z)=\log T(z)$. This leads to the theorem:

Theorem IIa. Among all univalent functions $f(z)$ in $D$ vanishing at $u$ and having a simple pole with residue 1 at $v$ and a prescribed value $\left|f^{\prime}(u)\right|$, the function $\exp \{Q(z ; u, v)+\alpha P(z ; u, v)\}$, for a suitable choice of $\alpha$, maps $D$ upon the domain whose complement has the maximum logarithmic area.

These results are now closely related to an extremum problem of quite different type. Consider for this purpose the class $\sqrt{ }$ of all functions which are regular and single-valued in $D$, vanish at $v$ and have the value 1 at $u$. A typical function of this class is

$$
f_{0}(z)=P(z ; u, v) \cdot P(u ; u, v)^{-1}
$$

Every other function of the class may be written in the form

$$
f(z)=f_{0}(z)+\phi(z)
$$

and, by definition, we have

$$
\phi(u)=\phi(v)=0 .
$$

Computing now the norm of $f(z)$, we find because of (39), (14) and (40')

$$
\left(f, f^{+}\right)=\pi P(u ; u, v)^{-1}+\left(\phi, \phi^{+}\right) .
$$

Since $\phi$ is regular and single-valued in $D$, its norm is non-negative and we arrive at the inequality

$$
\left(f, f^{+}\right) \geqq \pi P(u ; u, v)^{-1},
$$

valid for every function $f(z)$ of our class. Equality holds only for the function $f_{0}(z)$. This leads to the result:

THEOREM III. All functions $f(z)$ which are regular and single-valued in $D$ and satisfy $f(u)=1$ and $f(v)=0$ map this domain upon an area which is at least $\pi P(u ; u, v)^{-1}$. The extremum map is obtained by means of the function (39).

Comparing theorems Ia and III we obtain the interesting result:

THEOREM IIIa. For every domain $D$ the product between the maximal logarithmic area of the complement and the minimal area under mappings of the family S is exactly $\pi^{2}$.

There is even a simple geometric relationship between the extremum domains for the two different mapping problems. In fact, we find in view of (16) that the boundary curves of our second extremum problem are similar to the 
reflected logarithmic images of the boundary curves occurring in our initial extremum problem. They are, therefore, simple nonintersecting closed curves, which shows that the extremum problem with respect to the class $\mathfrak{\subseteq}$ leads to a domain which is at most $n$ times covered and that $P(z ; u, v)$ is at most $n$-valent. We state, therefore, the following theorem.

THEOREM IV. The function $Q(z ; u, v)$ is the logarithm of a univalent function and has logarithmic poles at $u$ and $v$; the function $P(z ; u, v)$ is regular and at most $n$-valued, if $n$ is the connectivity of the domain.

It will be of interest to us to have also the following result. Let $f_{i j}(z)$ be a map of $D$ on an annullus cut along concentric circular slits such that $C_{i}$ and $C_{j}, i \neq j$, go respectively into the inner and outer boundaries of the annullus, and let $g_{i j}(z)$ be a map of $D$ on an annullus cut along radial slits such that $C_{i}$ and $C_{j}, i \neq j$, go respectively into the inner and outer boundaries. Let $\mathfrak{F}_{i j}$ be the general class of schlicht maps $h_{i j}(z)$ of $D$ upon domains in annulli such that $C_{i}$ and $C_{j}$ are carried into the concentric circles bounding the annullus, and let $A_{h}$ denote the logarithmic area of the continua inside the images of the $C_{k}, i \neq k \neq j$. Then for prescribed modulus $\rho$ of the annullus, where $\rho$ lies between the moduli corresponding to $f_{i j}$ and $g_{i j}, A_{h}$ is maximized by the schlicht map $h_{i j}=\left(f_{i j} g_{i j}\right)^{1 / 2}\left(g_{i j} / f_{i j}\right)^{\lambda / 2}$, for suitable $\lambda,-1 \leqq \lambda \leqq 1$. If we pose the same problem without restriction on $\rho$ the extremal function is $\left(f_{i j} g_{i j}\right)^{1 / 2}$ and $\lambda=0$. This extremal problem plays for the harmonic measures the role which those we have considered in detail play for Green's and Neumann's functions, for, as we shall see later, $\log f_{i j}$ is a linear combination of harmonic measures. We skip the proof of the result, since it is so much like those already given.

Finally, we remark that by means of $P(z ; u, v)$ and $Q(z ; u, v)$ it is possible to obtain interesting mapping functions in the following way: We have, in view of (16), on every boundary continuum $C_{\nu}$

$$
P^{\prime}(z ; u, v) z^{\prime}=-\left(Q^{\prime}(z ; u, v) z^{\prime}\right)^{+}, \quad z^{\prime}=d z / d s .
$$

This shows that the quotient

$$
E(z ; u, v)=P^{\prime}(z ; u, v) / Q^{\prime}(z ; u, v)
$$

has on $C$ always the modulus 1 . Since $Q^{\prime}(z ; u, v) \neq 0$ for $z \in D, E(z ; u, v)$ maps $D$ upon the unit circle, of course covered several times. It is easy to determine the number of coverings. In fact, from (42) we derive that

$$
P^{\prime}(z ; u, v) Q^{\prime}(z ; u, v) z^{\prime 2}<0,
$$

By the argument principle we conclude, therefore, that the difference between the number of zeros and the number of poles of the left-hand product is $2 n-4$. Now, $Q^{\prime} \neq 0$ in $D$, but has two poles at $z=u$ and $z=v$; hence $P^{\prime}(z ; u, v)$ has $2 n-2$ zeros and the function $E(z ; u, v)$ maps $D$ upon the unit circle covered 
$2 n$ times. The close relations between functions which have the modulus 1 on $C$ and the domain functions $P(z ; u, v)$ and $Q(z ; u, v)$ will be discussed later.

3 . The method of contour integration. In this section systematic use will be made of the equation (16), which connects the functions $P(z ; u, v)$ and $Q(z ; u, v)$ at the boundary $C$ of $D$. It will be seen that this relation leads by means of the residue theorem to a number of important identities. The idea of procedure will become clear from our first application, which will, therefore, be carried out in more detail than the following ones.

We have by Cauchy's integral theorem

$$
\frac{1}{2 \pi i} \oint_{C} P(z ; u, v) P^{\prime}(z ; a, b) d z=0, \quad u, v, a, b \in D
$$

since $P(z ; u, v)$ and $P(z ; a, b)$ are regular in $D$. By means of (16) we may now replace in this contour integral $P$ by $Q$; taking into consideration that $Q(z ; a, b)$ does not change if $z$ describes a continuum $C_{\nu}$, that is,

$$
\oint_{c_{\nu}} Q^{\prime}(z ; a, b) d z=0
$$

we obtain from (45), (16) and (46)

$$
\frac{1}{2 \pi i} \oint_{c} Q(z ; u, v) Q^{\prime}(z ; a, b) d z=0 .
$$

The function $Q(z ; u, v)$ is not single-valued in $D$, but is so in the domain $D_{\gamma}$ obtained from $D$ by performing a cut along a smooth curve $\gamma$ from $u$ to $v$. Applying now the residue theorem to $D_{\gamma}$ and using equation (47), we find

$$
Q(a ; u, v)-Q(b ; u, v)+\frac{1}{2 \pi i} \int_{u}^{v} 2 \pi i Q^{\prime}(z ; a, b) d z=0,
$$

which leads to the symmetry rule

$$
Q(a ; u, v)-Q(b ; u, v)=Q(u ; a, b)-Q(v ; a, b) .
$$

This rule may be compared to the rule of interchange of parameter and argument in the theory of integrals on Riemann surfaces. It shows that $Q(u ; a, b)$ $-Q(v ; a, b)$ is analytic in all four variables, a result which we obtained in a different way at the end of $\S 1$.

For the next application we start with the identity

$$
\frac{1}{2 \pi i} \oint_{C} P(z ; u, v) Q^{\prime}(z ; a, b) d z=P(a ; u, v)-P(b ; u, v)
$$

which is an immediate consequence of the residue theorem and the analytic character of $P$ and $Q$ in $D$. By means of (16) we obtain 


$$
\begin{aligned}
P(a ; u, v)-P(b ; u, v) & =\frac{1}{2 \pi i} \oint_{c}\left(Q(z ; u, v) P^{\prime}(z ; a, b) d z\right)^{+} \\
& =\left(\frac{1}{2 \pi i} \oint_{C} P(z ; a, b) Q^{\prime}(z ; u, v) d z\right)^{+} ;
\end{aligned}
$$

a new application of the residue theorem leads to the symmetry law

$$
P(a ; u, v)-P(b ; u, v)=(P(u ; a, b))^{+}-(P(v ; a, b))^{+} .
$$

This result shows that $P(u ; a, b)-P(v ; a, b)$ is analytic in $u$ and $v$, but antianalytic in $a$ and $b$.

It is now clear how important pairs of functions are which have on $C$ conjugate boundary values. It is possible to define another pair of functions with this property and to obtain relations between them and the functions $P$ and $Q$. We start with two functions $A(z ; u)$ and $B(z ; u)$ which are both univalent in $D$ and are there regular except for a simple pole at $z=u$ with residue 1 . $A(z ; u)$ maps $D$ upon the complex plane slit along rectilinear segments parallel to the real axis, while $B(z ; u)$ maps $D$ upon the complex plane slit along rectilinear segments parallel to the imaginary axis. In both cases, the point $u$ corresponds to infinity. Our requirements determine the functions $A(z ; u)$ and $B(z ; u)$ up to an additive constant [5].

One has by definition

$$
A(z ; u)=\gamma_{\nu}(z)+i \alpha_{\nu}, \quad z \in C_{\nu},
$$

where $\gamma_{\nu}$ and $\alpha_{\nu}$ are real and depend on $u$, but $\alpha_{\nu}$ does not depend on $z$. In the same way

$$
B(z ; u)=\beta_{\nu}+i \delta_{\nu}(z), \quad z \in C_{\nu},
$$

with real $\beta_{\nu}$ and $\delta_{\nu}(z)$ and $\beta_{\nu}$ independent of $z$.

In view of preceding considerations it is suggestive to define now the pair of functions

$$
M(z ; u)=[A(z ; u)-B(z ; u)] / 2
$$

and

$$
N(z ; u)=[A(z ; u)+B(z ; u)] / 2 .
$$

Obviously, $M(z ; u)$ is regular everywhere in $D$ while $N(z ; u)$ has at $z=u$ a simple pole with residue 1 . Because of (53) and (54) one has for $z \in C_{v}$

$$
M(z ; u)=(N(z ; u))^{+}+\left(l_{v}(u)\right)^{+},
$$

where $l_{\nu}(u)=-i \alpha_{\nu}-\beta_{\nu}$ is a constant depending on $\nu$ and (not analytically) on $u$.

The functions $M(z ; u)$ and $N(z ; u)$ play an important role in the theory of conformal mapping. One shows by the methods of $\S 2$ that $N(z ; u)$ is univalent 
in $D$ and that even every combination

$$
F(z ; u)=N(z ; u)+\lambda M(z ; u),
$$$$
0 \leqq \lambda \leqq 1,
$$

is univalent in $D$. By means of these functions the following extremum problems can be solved:

I. Consider the family of all functions which are regular and single-valued in $D$ and have at $u \in D$ the derivative 1 . Determine that function of the family which maps $D$ upon a domain of smallest area.

The solution of this problem is given by the function $M(z ; u) M^{\prime}(u ; u)^{-1}$ (where $M^{\prime}(z ; u)=d M(z ; u) / d z$ ). The minimum area is $\pi M^{\prime}(u ; u)^{-1} . M(z ; u)$ is at most $n$-valent in $D$, as can be shown by reasoning similar to that in $\$ 2$.

II. Consider the family of all functions which are univalent and singlevalued in $D$ and have at the point $u \in D$ a simple pole with residue 1 . Each such function maps $D$ upon a domain $\Delta$; we seek a function of this class which yields a maximum area for the complement $C(\Delta)$ of $\Delta$ in the complex plane.

The solution of this problem is given by the function $N(z ; u)[11,14]$. The maximum area is $\pi M^{\prime}(u ; u)$. We have again the remarkable fact that the minimum area of problem I and the maximum area of problem II have for every domain $D$ the product $\pi^{2}[13]$.

III. Consider the family of all functions which are univalent and singlevalued in $D$ and have at the point $u \in D$ a development

$$
f(z)=(z-u)^{-1}+k_{0}+k_{1}(z-u)+\cdots,
$$

with fixed $\operatorname{Re}\left\{k_{1}\right\}$. Each such function maps $D$ upon a domain $\Delta$; we seek a function of this class which yields a maximum area for the complement $C(\Delta)$ of $\Delta$ (cf. [3]).

The solution is given by that function (58) which has the right value of $\operatorname{Re}\left\{k_{1}\right\}$. The great analogy between the pairs $M, N$ and $P, Q$ is evident. We shall now show that they are in fact closely related, and this can again be done by our integration method.

Starting with the identity

$$
\frac{1}{2 \pi i} \oint_{c} M(z ; a) P^{\prime}(z ; u, v) d z=0, \quad a, u, v \in D,
$$

and applying the relations (16) and (57), we obtain easily

$$
\frac{1}{2 \pi i} \oint_{C} N(z ; a) Q^{\prime}(z ; u, v) d z=0
$$

By virtue of the residue theorem we arrive finally at

$$
Q^{\prime}(a ; u, v)=N(v ; a)-N(u ; a),
$$

connecting the function $N(z ; u)$ with the derivative of the function $Q(z ; u, v)$. We recognize, in particular, the fact that $Q^{\prime}(z ; u, v)$ is analytic in all three 
arguments. This might also have been expected from (49) for the limit case $a \rightarrow b$.

Let us consider next the following instance of the residue theorem:

$$
\frac{1}{2 \pi i} \oint_{c} N(z ; a) P^{\prime}(z ; u, v) d z=P^{\prime}(a ; u, v), \quad a, u, v \in D .
$$

From (16) and (57) we conclude

$$
\frac{1}{2 \pi i} \oint_{C} M(z ; a) Q^{\prime}(z ; u, v) d z=\left(P^{\prime}(a ; u, v)\right)^{+},
$$

and applying again the residue theorem, we obtain

$$
\left(P^{\prime}(a ; u, v)\right)^{+}=M(u ; a)-M(v ; a) .
$$

This shows that $P^{\prime}(z ; u, v)$ is analytic in $z$ and anti-analytic in $u$ and $v$.

Let us combine further $M$ and $N$ in the following equation:

$$
\frac{1}{2 \pi i} \oint_{c} M(z ; u) N^{\prime}(z ; v) d z=-M^{\prime}(v ; u), \quad\left\{\begin{array}{l}
M^{\prime}(z ; u)=\frac{d}{d z} M(z ; u) \\
N^{\prime}(z ; u)=\frac{d}{d z} N(z ; u)
\end{array}\right.
$$

This leads by virtue of (57) to

$$
\frac{1}{2 \pi i} \oint_{C} N(z ; u) M^{\prime}(z ; v) d z=\left(M^{\prime}(v ; u)\right)^{+},
$$

and, by means of the residue theorem, to the symmetry law

$$
M^{\prime}(u ; v)=\left(M^{\prime}(v ; u)\right)+
$$

From the equation

$$
\frac{1}{2 \pi i} \oint_{C} M(z ; u) M^{\prime}(z ; v) d z=0, \quad u, v \in D
$$

we derive in the same way

$$
\frac{1}{2 \pi i} \oint_{c} N(z ; u) N^{\prime}(z ; v) d z=0,
$$

which leads again by the residue theorem to the analogous symmetry formula for $N$,

$$
N^{\prime}(v ; u)=N^{\prime}(u, v) \text {. }
$$

From (65) and (67) we infer that $M^{\prime}(u ; v)$ is analytic in $u$ and anti-analytic in $v$, while $N^{\prime}(u ; v)$ is symmetric and analytic in both arguments. 
One recognizes again the great similarity between the above relations and the corresponding formulas in the theory of Abelian integrals. The functions $P$ and $Q$ correspond to the fundamental integrals of the third kind and $M$ and $N$ to the integrals of the second kind. The functions $P$ and $M$, however, have no singularities at all in $D$. This is closely related to the result of Schottky [15] that a domain $D$ of connectivity $n$ corresponds to a half of a symmetric Riemann surface of genus $n-1$. $P$ and $M$ correspond, therefore, to fundamental integrals having their singularity in the missing half of the Riemann surface. Our method of integration along the boundary $C$ of $D$ is closely related to a procedure of Riemann for obtaining relations between Abelian integrals; it has been called the method of contour integration. The central role of the formulas (16) and (57) in this type of reasoning is obvious.

Pursuing the analogy between domain functions of a domain $D$ and Abelian integrals on a Riemann surface, we introduce now functions which correspond to Abelian integrals of the first kind. For this purpose we define the harmonic measures of each boundary continuum $C_{\nu}$ at the point $z$ with respect to the domain $D$. These functions $\omega_{\nu}(z)$, which play an important role in the general theory of functions, are defined as follows:

$\omega_{\nu}(z)$ is harmonic for $z \in D$ and has on $C_{\mu}$ the boundary values $\delta_{\mu \nu}$. One has obviously the relation

$$
\sum_{\nu=1}^{n} \omega_{\nu}(z)=1,
$$

since the left-hand side represents a function harmonic in $D$ with boundary values 1 on $C$.

We may complete $\omega_{\nu}(z)$ to an analytic function $w_{\nu}(z)$ such that

$$
\omega_{\nu}(z)=\operatorname{Re}\left\{w_{\nu}(z)\right\} .
$$

$w_{\nu}(z)$ is defined up to an additive imaginary constant; it is, in general, not single-valued in $D$, though it has a single-valued real part. If $z$ describes a circuit around the contour $C_{\mu}, w_{\nu}(z)$ increases by the period

$$
\oint_{c_{\mu}} w_{\nu}^{\prime}(z) d z=-i \oint_{C_{\mu}} \frac{\partial \omega_{\nu}}{\partial n} d s=-2 \pi i P_{\mu \nu},
$$

where $\partial / \partial n$ denotes differentiation in the direction of the interior normal at $z \in C$.

Since the real part of $w_{\nu}(z)$ is constant on each boundary continuum $C_{\mu}$ of $C$, we have

$$
w_{\nu}^{\prime}(z) d z=\text { imaginary for } z \text { varying on } C .
$$

This property will now be used in contour integration in the same way as (16) and (57). 
Consider the integral

$$
\frac{1}{2 \pi i} \oint_{C} P(z ; u, v) w_{v}^{\prime}(z) d z=0, \quad u, v \in D
$$

We may, in fact, apply Cauchy's theorem, since $w_{p}^{\prime}(z)$ is single-valued and regular in $D$. Applying now (16) and (71), we obtain

$$
\frac{1}{2 \pi i} \sum_{\mu=1}^{n} \oint_{c_{\mu}}\left[Q(z ; u, v)-k_{\mu}(u, v)\right] w_{\nu}^{\prime}(z) d z=0 .
$$

The term $k_{\mu}(u, v)$ in (16) cannot be neglected now, since $w_{\nu}^{\prime}(z)$ is not the derivative of a single-valued function. Using (70), we may put (73) into the form

$$
\frac{1}{2 \pi i} \oint_{C} Q(z ; u, v) w_{\nu}^{\prime}(z) d z=-\sum_{\mu=1}^{n} k_{\mu}(u, v) P_{\mu \nu}
$$

The left-hand integral may be evaluated as before by applying Cauchy's theorem to a domain $D_{\gamma}$ obtained from $D$ by making a cut from $u$ to $v$ along a smooth curve $\gamma \cdot Q(z ; u, v)$ is single-valued in $D_{\gamma}$ and has at the two borders of the cut a saltus of amount $2 \pi i$. Hence, we find

$$
w_{\nu}(u)-w_{\nu}(v)=\sum_{\mu=1}^{n} k_{\mu}(u, v) P_{\mu \nu}, \quad \nu=1,2, \cdots, n,
$$

which shows an important relation between the constants $k_{\mu}$ appearing in (16) and the harmonic measures.

In the same fashion, we derive from

$$
\frac{1}{2 \pi i} \oint_{C} M(z ; u) w_{\nu}^{\prime}(z) d z=0, \quad u \in D
$$

and from (57), (71)

$$
\frac{1}{2 \pi i} \sum_{\mu=1}^{n} \oint_{C_{\mu}}\left[N(z ; u)+l_{\mu}(u)\right] w_{\nu}^{\prime}(z) d z=0 .
$$

This leads because of (70) to

$$
w_{\nu}^{\prime}(u)=\sum_{\mu=1}^{n} l_{\mu}(u) P_{\mu \nu},
$$

a relation connecting the constants $l_{\mu}$ occurring in (57) with the derivatives of the harmonic measures.

Let us point out finally one interesting property of the harmonic measures with respect to the scalar product $\left(f, g^{+}\right)$defined in $\$ 1$. Consider, for this purpose, the scalar product 


$$
\left(f, w_{\nu}^{+}\right)=\iint_{D} f^{\prime}(z)\left(w_{\nu}^{\prime}(z)\right)^{+} d x d y
$$

between $w_{\nu}(z)$ and an arbitrary function $f(z)$ of the class $\Omega$. Integrating by parts, we obtain

$$
\left(f, w_{\nu}^{+}\right)=-\frac{1}{2 i} \oint_{C} f(z)\left(w_{\nu}^{\prime}(z) d z\right)^{+}=\frac{1}{2 i} \oint_{C} f(z) w_{\nu}^{\prime}(z) d z
$$

in view of (71). But the last integral vanishes because of Cauchy's theorem. Thus, we see that

$$
\left(f, w_{\nu}^{+}\right)=0 \quad \text { for every } f(z) \in \Omega,
$$

that is, the functions $w_{\nu}(z)$ are orthogonal to all functions $f(z) \in \Omega$.

In order to compute the expressions

$$
\left(w_{\nu}, w_{\mu}^{+}\right)=\iint_{D} w_{\nu}^{\prime}(z)\left(w_{\mu}^{\prime}(z)\right)+d x d y
$$

we have to perform a slightly different method of partial integration. We find

$$
\left(w_{\nu}, \dot{w}_{\mu}^{+}\right)=-\frac{1}{i} \oint_{C} \omega_{\nu}(z)\left(w_{\mu}^{\prime}(z) d z\right)^{+}=\frac{1}{i} \oint_{C_{\nu}} w_{\mu}^{\prime}(z) d z=-2 \pi P_{\mu \nu}
$$

This shows that the terms $\left(w_{\nu}, w_{\mu}^{+}\right)$are real and form, therefore, a symmetric matrix. The $P_{\mu \nu}$ form a symmetric matrix belonging to a semi-definite quadratic form. In fact, consider the analytic function

$$
w(z)=\sum_{\nu=1}^{n} \xi_{\nu} w_{\nu}(z)
$$

with real, but otherwise arbitrary, coefficients $\xi_{\nu}$. Its real part is single-valued in $D$ and has on $C_{\nu}$ the boundary values $\xi_{\nu}$. Since, in view of (83),

$$
\left(w, w^{+}\right)=\iint_{D}\left|w^{\prime}(z)\right|^{2} d x d y=-2 \pi \sum_{\mu, v=1}^{n} \xi_{\mu} \xi_{\nu} P_{\mu \nu},
$$

and this is a non-negative expression, our assertion is proved. The quadratic form may vanish only if $\left|w^{\prime}(z)\right| \equiv 0$, that is, if $w(z)$ is constant. But this is possible only if all $\xi_{\nu}$ are equal. In this case, $w(z)$ is really a constant because of (68).

Another way of understanding the fact that

$$
\sum_{\mu, \nu=1}^{n} P_{\mu \nu}=0
$$

is the consideration of the periods of 


$$
\sum_{\nu=1}^{n} w_{\nu}(z)=\text { const. }
$$

for circuits around the boundary continuum $C_{\mu}$. One finds in view of (70)

$$
\sum_{\nu=1}^{n} P_{\mu \nu}=0, \quad \quad \mu=1,2, \cdots, n,
$$

which leads immediately to (86).

It should be remarked that a system of equations of the form (75) or (78) determines the unknowns $k_{\mu}$ or $l_{\mu}$ only up to a common additive constant. In fact, if $k_{\mu}(\mu=1,2, \cdots, n)$ is a solution of equations (75), then $k_{\mu}$ $+k(\mu=1, \cdots, n)$ is, for arbitrary $k$, another solution in view of $\left(87^{\prime}\right)$. On the other hand, it follows from the semi-definite character of the matrix $\left(P_{\mu \nu}\right)$ that this is the most general solution of the system of equations (75).

Let us investigate briefly the geometric meaning of the relation (75). It is evident from the definition of the $P_{\mu \nu}$ that when $u$ makes a circuit about the $\mu$ th boundary contour $C_{\mu}$ of $D, k_{\nu}(u, v)$ has a period

$$
-2 \pi i \delta_{\mu \nu}+\frac{2 \pi i}{n}=\oint_{C_{\mu}} \frac{d k_{\nu}(u, v)}{d u} d u .
$$

Hence the function

$$
K_{\mu \nu}(u, v)=k_{\mu}(u, v)-k_{\nu}(u, v)
$$

has about $C_{i}, \mu \neq i \neq \nu$, no period, and about $C_{\nu}$ and $C_{\mu}$ the periods $2 \pi i$ and $-2 \pi i$ respectively. Hence we see in the usual fashion that $\exp \left\{k_{\mu}(u, v)\right.$ $\left.-k_{\nu}(u, v)\right\}$ is a map $f_{\mu \nu}(u)$ of $D$ upon an annullus cut along concentric circular slits, such as we introduced in $\$ 2$. Since the mapping function $f_{\mu \nu}(u)$ is determined up to a constant factor, we conclude further that $K_{\mu \nu}(u, v)$ has the form

$$
K_{\mu \nu}(u, v)=K_{\mu \nu}^{(1)}(u)-K_{\mu \nu}^{(1)}(v)+\text { const. },
$$

a result which is also obvious from (75).

4. On Green's and Neumann's functions. We introduce now the two domain functions which play a central role in the theory of logarithmic potential of plane domains.

(a) Green's function $g(z ; \zeta)$ is harmonic for $z \in D$ except for the point $\zeta \in D$, where $g(z ; \zeta)+\log |z-\zeta|$ is harmonic. If $z$ converges to the boundary $C$ of $D$, Green's function tends to zero.

Green's function plays a decisive role in the first boundary value problem for harmonic functions. It is well known that under our assumption on $D$ Green's function is still continuously differentiable on the boundary $C$ of $D$. It satisfies the symmetry law 


$$
g(z ; \zeta)=g(\zeta ; z)
$$

and is, therefore, also harmonic in $\zeta$ except for $\zeta=z$.

(b) Neumann's function $\gamma(z ; \zeta)$ is harmonic for $z \in D$ except for the point $\zeta \in D$, where $\gamma(z ; \zeta)+\log |z-\zeta|$ is harmonic. $\gamma(z ; \zeta)$ is continuously differentiable in $D+C$ and on $C$ one has

$$
\frac{\partial \gamma(z ; \zeta)}{\partial n}=\frac{2 \pi}{L}
$$

where $L$ is the total length of the boundary curves $C$. Finally one normalizes Neumann's function by the requirement

$$
\oint_{c} \gamma(z ; \zeta) d s=0
$$

Neumann's function serves for solving the second boundary value problem for harmonic functions. Again one has a symmetry law

$$
\gamma(z ; \zeta)=\gamma(\zeta ; z)
$$

It is useful to complete the harmonic functions $g(z ; \zeta)$ and $\gamma(z ; \zeta)$ to analytic functions of $z$. For this purpose, we introduce two functions $p(z ; \zeta)$ and $\pi(z ; \zeta)$ analytic in $z$ and such that

$$
g(z ; \zeta)=\operatorname{Re}\{p(z ; \zeta)\}, \quad \gamma(z ; \zeta)=\operatorname{Re}\{\pi(z ; \zeta)\}
$$

These requirements fix $p$ and $\pi$ only up to an additive imaginary constant which may still depend on $\zeta$. Both functions have obviously a logarithmic pole at $z=\zeta$; they are not single-valued in $D$. Besides the period $2 \pi i$ caused by the logarithmic pole they have periods with respect to circuits of $z$ around the boundary continua $C_{\nu}$. In fact, in view of the Cauchy-Riemann equations we have

$$
\oint_{c_{\nu}} d p(z ; \zeta)=i \oint_{c_{\nu}} \frac{\partial}{\partial s} \operatorname{Im}\{p(z ; \zeta)\} d s=-i \oint_{c_{\nu}} \frac{\partial g(z ; \zeta)}{\partial n} d s
$$

Now, obviously

$$
\omega_{\nu}(\zeta)=\frac{1}{2 \pi} \oint_{C_{\nu}} \frac{\partial g(z ; \zeta)}{\partial n} d s
$$

is that harmonic function of $\zeta$ which has on the boundary continuum $C_{\mu}$ the value $\delta_{\mu \nu}$ and is the harmonic measure of $C_{\nu}$ with respect to $D$ at the point $\zeta$, as defined in the last section. Hence, we may put (93) into the form

$$
\oint_{C_{\nu}} d p(z ; \zeta)=-2 \pi i \omega_{\nu}(\zeta)
$$


The periods of $\pi(z ; \zeta)$ follow from the expression

$$
\oint_{C_{\nu}} d \pi(z ; \zeta)=i \oint_{c_{\nu}} \frac{\partial}{\partial s} \operatorname{Im}\{\pi(z ; \zeta)\} d s=-i \oint_{c_{\nu}} \frac{\partial \gamma(z ; \zeta)}{\partial n} d s
$$

In view of (89) this yields

$$
\oint_{C_{\nu}} d \pi(z ; \zeta)=-2 \pi i \frac{L_{\nu}}{L}
$$

where $L_{\nu}$ is the length of the boundary continuum $C_{\nu}$. It is important to note that the periods of $\pi(z ; \zeta)$ do not depend on $\zeta$ and that the function

$$
q(z ; v, \zeta)=\pi(z ; v)-\pi(z ; \zeta)
$$

is, therefore, free from periods around the $C_{\nu}$.

It is easily seen from (89) that the real part of $q(z ; v, \zeta)$ has on $C$ the normal derivative zero and its imaginary part is constant along each $C_{\boldsymbol{v}}$. Hence, one concludes by the usual reasoning that $\exp \{q(z ; v, \zeta)\}$ is a single-valued function which maps $D$ univalently upon the entire plane slit along rectilinear segments pointing towards the origin. The points $\zeta$ and $v$ correspond to the origin and infinity respectively. Hence, this function is related to $\Psi(z ; \zeta, v)$, defined in $\$ 1$, by an equation

$$
\log \Psi(z ; \zeta, v)=\pi(z ; v)-\pi(z ; \zeta)+\text { const. }
$$

The additive constant in (99) may be easily determined from the normalization of $\Psi(z ; \zeta, v)$ to have the residue 1 at its simple pole $v$.

For our further developments it is important to notice that $d p(z ; \zeta) / d z$ $=p^{\prime}(z ; \zeta)$ and $d \pi(z ; \zeta) / d z=\pi^{\prime}(z ; \zeta)$ are single-valued in $D$ and have a simple pole with residue -1 at $\zeta$. On the boundary $C$ we have evidently

$$
p^{\prime}(z ; \zeta) d z=\text { imaginary, }
$$

$z$ varying on $C$,

since the real part of $p(z ; \zeta)$ is zero there. On the other hand, we obtain from the Cauchy-Riemann equations

$$
\begin{aligned}
\pi^{\prime}(z ; \zeta) d z=\frac{\partial \pi(z ; \zeta)}{\partial s} d s & =\left[\frac{\partial \gamma(z ; \zeta)}{\partial s}-i \frac{\partial \gamma(z ; \zeta)}{\partial n}\right] d s \\
& =\left[\frac{\partial \gamma(z ; \zeta)}{\partial s}-\frac{2 \pi i}{L}\right] d s
\end{aligned}
$$

This leads to the remarkable relation

$$
\left(\pi^{\prime}(z ; \zeta) d z\right)^{+}=\pi^{\prime}(z ; \zeta) d z+\frac{4 \pi i}{L} d s
$$

After these preparations we apply the method of contour integration to 
$p(z ; \zeta)$ and $\pi(z ; \zeta)$. We start with the identity

$$
\frac{1}{2 \pi i} \oint_{C} P(z ; u, v) p^{\prime}(z ; \zeta) d z=-P(\zeta ; u, v), \quad u, v, \zeta \in D
$$

We transform the left-hand integral by means of (16) and (100); we obtain

$$
\frac{1}{2 \pi i} \sum_{\mu=1}^{n} \oint_{C_{\mu}}\left[Q(z ; u, v)-k_{\mu}(u, v)\right] p^{\prime}(z ; \zeta) d z=(P(\zeta ; u, v))^{+}
$$

Because of the period-formula (95), we find

$$
\frac{1}{2 \pi i} \oint_{c} Q(z ; u, v) p^{\prime}(z ; \zeta) d z+\sum_{\mu=1}^{n} k_{\mu}(u, v) \omega_{\mu}(\zeta)=(P(\zeta ; u, v))^{+}
$$

Now, we evaluate the left-hand integral by applying Cauchy's theorem to the domain $D_{\gamma}$ which is obtained by slitting $D$ along a smooth curve $\gamma$ from $u$ to $v$. We find easily

$$
p(v ; \zeta)-p(u ; \zeta)=Q(\zeta ; u, v)+(P(\zeta ; u, v))^{+}-\sum_{\mu=1}^{n} k_{\mu}(u, v) \omega_{\mu}(\zeta)
$$

This formula expresses $p(v ; \zeta)$ in terms of the functions $P$ and $Q$; it is clear that by our definition only a formula for $p(v ; \zeta)-p(u ; \zeta)$ and not for $p(v ; \zeta)$ alone was to be expected. For our definition left an arbitrary imaginary constant free which might depend on $\zeta$. Hence, only a difference between $p(v ; \zeta)$ and its value at a fixed point $u \in D$ is uniquely defined; this shows that (103) yields the maximum information which is to expected for $p(v ; \zeta)$.

We apply now the same considerations to the function $\pi(z ; \zeta)$. From

$$
\frac{1}{2 \pi i} \oint_{c} P(z ; u, v) \pi^{\prime}(z ; \zeta) d z=-P(\zeta ; u, v), \quad u, v, \zeta \in D
$$

we obtain by means of (16) and (101')

$$
\begin{aligned}
\frac{1}{2 \pi i} \sum_{\mu=1}^{n} \oint_{c_{\mu}}\left[Q(z ; u, v)-k_{\mu}(u, v)\right]\left[\pi^{\prime}(z ; \zeta) z^{\prime}+\frac{4 \pi i}{L}\right] d s & \\
& =-(P(\zeta ; u, v))^{+}, \quad z^{\prime}=\frac{d z}{d s}
\end{aligned}
$$

Hence, applying the period formula (97) we find after a simple transformation

$$
\begin{aligned}
\frac{1}{2 \pi i} \oint_{C} Q(z ; u, v) \pi^{\prime}(z ; \zeta) d z-\sum_{\mu=1}^{n} k_{\mu}(u, v) \frac{L_{\mu}}{L}+\frac{2}{L} \oint_{C} Q(z ; u, v) d s \\
=-(P(\zeta ; u, v))^{+}
\end{aligned}
$$

Using again Cauchy's formula with respect to $D_{\gamma}$, we arrive finally at the 
equation

$$
\begin{aligned}
\pi(v ; \zeta)-\pi(u ; \zeta)=Q(\zeta ; u, v) & -(P(\zeta ; u, v))^{+} \\
& +\sum_{\mu=1}^{n} k_{\mu}(u, v) \frac{L_{\mu}}{L}-\frac{2}{L} \oint_{C} Q(z ; u, v) d s,
\end{aligned}
$$

which expresses $\pi(v ; \zeta)$ in terms of the functions $Q$ and $P$.

At this stage it becomes evident that we may obtain much simpler formulas by an appropriate change of normalization of the functions $P(z ; u, v)$ and $Q(z ; u, v)$. These functions were defined as the difference and the sum of the logarithms of two canonical mapping functions. The geometrical properties of these two mappings, namely mapping upon a circular or radial slit domain, determine the two functions only up to a constant factor. We determined this factor by the requirement that the residue at the pole $z=v$ be one. This normalization was quite natural in connection with the extremum problems of $\S 2$, which made a distinction between $u$ and $v$. In our formal transformations of $\$ \S 3$ and 4 , however, we made use only of the equations (16), and not of the requirements at the point $z=v$. From the normalization (90), which fixed an arbitrary additive constant without distinction of special points, and, in particular, from (105), we recognize that a new type of normalization is preferable and will lead to much simpler expressions for Neumann's function. In fact, we shall add to $P(z ; u, v)$ and $Q(z ; u, v)$ constant terms with respect to $z$ which depend, however, on $u$ and $v$ such that

$$
\oint_{c} P(z ; u, v) d s_{z}=\oint_{C} Q(z ; u, v) d s_{z} \equiv 0 \quad \text { for every } u, v \in D .
$$

We denote the renormalized functions again by $P(z ; u, v)$ and $Q(z ; u, v)$ in order to avoid an excessive number of letters; from now on $P$ and $Q$ will denote only the fundamental domain functions with the new normalization (106).

It is clear that the new functions $P$ and $Q$ satisfy again equations (16), but with new constants $k_{\nu}(u, v)$. Because of (106) we derive from (16) for the $k_{v}(u, v)$ the relation

$$
\sum_{v=1}^{n} L_{\nu} k_{i}(u, v) \equiv 0, \quad u, v \in D .
$$

Returning to (105), which was proved from (16) and remains valid for the new functions $P$ and $Q$, we find by virtue of (106) and (107)

$$
\pi(v ; \zeta)-\pi(u ; \zeta)=Q(\zeta ; u, v)-(P(\zeta ; u, v))^{+}
$$

By means of (107) we are further able to invert the system of equations (75) for the $k_{\mu}(u, v)$ and to express these terms by means of $w_{\nu}(u)-w_{\nu}(v)$. In fact, taking the first $(n-1)$ equations (75) and eliminating $k_{n}(u, v)$ from 
them by means of (107) we find all other $k_{\mu}(u, v)$ as linear combinations of $w_{\nu}(u)-w_{\nu}(v)$. This shows that every $k_{\mu}(u, v)$ is a multiple-valued analytic function of $u$ and $v$.

Finally, let us solve the equations (103) and (108) with respect to $Q(\zeta ; u, v)$ and $(P(\zeta ; u, v))^{+}$; we obtain

$$
\begin{aligned}
Q(\zeta ; u, v)= & \frac{1}{2}[\{p(v ; \zeta)-p(u ; \zeta)\}+\{\pi(v ; \zeta)-\pi(u ; \zeta)\}] \\
& +\frac{1}{2} \sum_{\mu=1}^{n} k_{\mu}(u, v) \omega_{\mu}(\zeta), \\
(P(\zeta ; u, v))^{+}= & \frac{1}{2}[\{p(v ; \zeta)-p(u ; \zeta)\}-\{\pi(v ; \zeta)-\pi(u ; \zeta)\}] \\
& +\frac{1}{2} \sum_{\mu=1}^{n} k_{\mu}(u, v) \omega_{\mu}(\zeta) .
\end{aligned}
$$

These equations show that $Q(\zeta ; u, v)$ is analytic in $\zeta, u$ and $v$ and satisfies the symmetry law

$$
Q(\zeta ; u, v)=-Q(\zeta ; v, u)
$$

$P(\zeta ; u, v)$ is analytic in $\zeta$ and anti-analytic in $u, v$ and has the symmetry rule

$$
P(\zeta ; u, v)=-P(\zeta ; v, u) \text {. }
$$

The analytic behavior of $P(z ; u, v)$ in dependance on its arguments might also be understood from the following considerations: We had in $\$ 1$ to restrict the class $\Omega$ in order to exclude from this class all nonvanishing constants. We did this by distinguishing a point $v \in D$ and requiring the vanishing of all functions of our subclass $\Omega_{v}$ at $v$. Our new normalization for $P$ and $Q$ leads naturally to a new subclass of $\Omega$. Let $\Omega_{1}$ be the class of all functions $f(z) \in \Omega$ which satisfy the condition

$$
\oint_{c} f(z) d s=0
$$

We have, however, to give a meaning to the integral (113) for functions which are not defined on $C$. This is possible by the remark that for every function $f(z)$ which is continuously differentiable in $D+C$ we have in view of (101')

$$
\begin{aligned}
\left(f, \pi^{+}\right) & =-\frac{1}{2 i} \oint_{c} f(z)(d \pi(z ; \zeta))^{+} \\
& =-\frac{1}{2 i} \oint_{c} f(z) \pi^{\prime}(z ; \zeta) d z-\frac{2 \pi}{L} \oint_{c} f(z) d s,
\end{aligned}
$$

whence by virtue of the residue theorem 


$$
\oint_{C} f(z) d s=-\frac{L}{2 \pi}\left(f(z),(\pi(z ; \zeta))^{+}\right)+\frac{L}{2} f(\zeta) .
$$

The right-hand side of $\left(114^{\prime}\right)$ is defined for every function $f(z) \in \Omega$, since it may be expressed by an improper integral extended over $D$. This permits an interpretation of the condition (113) for every function $f(z) \in \Omega$ and a unique definition for the class $\Omega_{1}$. It is obvious that the only constant in this class is $f(z) \equiv 0$. We introduce now the metric (6) or (7) into this class and remark that $\left(f, f^{+}\right)=0$ implies, in $\Omega_{1}, f(z) \equiv 0$.

The function $P(z ; u, v)$ belongs to $\Omega_{1}$ because of its normalization (106). It satisfies still the relation (14) for every $f(z) \in \Omega_{1}$. We introduce now a complete orthonormal system $\left\{f_{\nu}(z)\right\}$ for the class $\Omega_{1}$, which is possible because of our exclusion of constants; developing $P(z ; u, v)$ into a Fourier series with respect to this system, we obtain because of (14)

$$
P(z ; u, v)=\pi \sum_{\nu=1}^{\infty} f_{\nu}(z)\left[\left(f_{v}(u)\right)^{+}-\left(f_{\nu}(v)\right)^{+}\right] .
$$

This series converges uniformly in each closed subdomain of $D$; it shows that $P$ is analytic in $z$ and anti-analytic in $u$ and $v$. The symmetry law (112) is also evident from (115).

Finally, we derive from (103) and (108) two remarkable identities. Differentiating (103) and (108) with respect to $v$ and $\zeta$ and comparing the results, we find

$$
\frac{\partial^{2} p}{\partial v \partial \zeta}=-\frac{\partial^{2} \pi}{\partial v \partial \zeta}-\frac{1}{2} \sum_{\mu=1}^{n} \frac{\partial}{\partial v} k_{\mu}(u, v) w_{\mu}^{\prime}(\zeta),
$$

while the same reasoning after differentiation with respect to $v$ and $\zeta^{+}$yields

$$
\frac{\partial^{2} p}{\partial v \partial \zeta^{+}}=\frac{\partial^{2} \pi}{\partial v \partial \zeta^{+}}-\frac{1}{2} \sum_{\mu=1}^{n} \frac{\partial}{\partial v} k_{\mu}(u, v)\left(w_{\mu}^{\prime}(\zeta)\right)^{+} .
$$

These two relations between the second derivatives of Green's and Neumann's functions play a role in the theory of the kernel function of an orthonormal system [2].

It is perhaps worth remarking at this point that many of our identities obtained by contour integration and the boundary relation (16) can be derived directly by inspection of the geometric character of the mappings involved. The relations (116) and (117) offer a good opportunity of demonstrating this fact. Indeed, let $\zeta=\sigma+i \tau$. The functions

$$
\begin{aligned}
& \alpha(v ; \zeta)=p(v ; \zeta)+\sum_{\mu=1}^{n} k_{\mu}(u, v) \omega_{\mu}(\zeta), \\
& \beta(v ; \zeta)=\pi(v ; \zeta)
\end{aligned}
$$


have fixed periods about each $C_{\nu}$ which are independent of $v, \zeta$. Hence, the derivatives with respect to $\sigma$ and $\tau$ will have no periods. Furthermore, since $\operatorname{Re}\{\alpha(v, \zeta)\}$ and $\operatorname{Im}\{\beta(v, \zeta)\}$ have at the worst variations along the $C_{\nu}$ which are independent of $\zeta$, the derivatives will have for $\alpha$ constant real part and for $\beta$ constant imaginary part on each $C_{\nu}$. Thus the partial derivatives $\partial \alpha / \partial \sigma$ and $-i \partial \beta / \partial \tau$ map $D$ on domains bounded by vertical slits, and $-i \partial \alpha / \partial \tau$ and $\partial \beta / \partial \sigma$ map $D$ on domains bounded by horizontal slits. We see, then, by the cancelation of the poles, that

$$
\begin{aligned}
& \frac{\partial p}{\partial \sigma}+\sum_{\mu=1}^{n} k_{\mu}(u, v) \frac{\partial \omega_{\mu}}{\partial \sigma}+i \frac{\partial \pi}{\partial \tau}=\text { const., } \\
& \frac{\partial p}{\partial \tau}+\sum_{\mu=1}^{n} k_{\mu}(u, v) \frac{\partial \omega_{\mu}}{\partial \tau}-i \frac{\partial \pi}{\partial \sigma}=\text { const. }
\end{aligned}
$$

But these relations imply (116), (117), upon differentiation with respect to $v$. This method often gives insight and shorter proofs, but because of its unsystematic nature we do not investigate it further, with one exception at the end of $\S 6$.

Now, we have to consider an appropriate normalization for the functions $M(z ; u)$ and $N(z ; u)$, which were defined in $\$ 3$ up to an additive constant. We require again the normalization

$$
\oint_{C} M(z ; u) d s_{z}=\oint_{C} N(z ; u) d s_{z} \equiv 0, \quad \text { for every } u \in D,
$$

which implies in view of (57)

$$
\sum_{\nu=1}^{n} L_{\nu} l_{\nu}(u) \equiv 0
$$$$
u \in D \text {. }
$$

The first advantage of this normalization is the fact that the $l_{\nu}(u)$ 's, which were until now defined by (78) only up to an additive constant, are defined in a unique way and become linear combinations of the $w_{\nu}^{\prime}(u)$, that is, analytic functions of $u$. Next, we may apply to $M(z ; u), N(z ; u), p(z ; \zeta)$ and $\pi(z ; \zeta)$ the method of contour integration and perform the same transformations as we did before with $P(z ; u, v)$ and $Q(z ; u, v)$. We obtain finally

$$
\begin{gathered}
p^{\prime}(z ; \zeta)=N(\zeta ; z)-(M(\zeta ; z))^{+}+\sum_{\nu=1}^{n} l_{\nu}(z) \omega_{\nu}(\zeta) \\
\pi^{\prime}(z ; \zeta)=N(\zeta ; z)+(M(\zeta ; z))^{+} .
\end{gathered}
$$

Solving these equations with respect to $N(\zeta ; z)$ and $(M(\zeta ; z))^{+}$, we obtain

$$
N(\zeta ; z)=\frac{1}{2}\left[\pi^{\prime}(z ; \zeta)+p^{\prime}(z ; \zeta)\right]-\frac{1}{2} \sum_{\nu=1}^{n} l_{\nu}(z) \omega_{\nu}(\zeta)
$$




$$
(M(\zeta ; z))^{+}=\frac{1}{2}\left[\pi^{\prime}(z ; \zeta)-p^{\prime}(z ; \zeta)\right]+\frac{1}{2} \sum_{\nu=1}^{n} l_{\nu}(z) \omega_{\nu}(\zeta),
$$

which shows that because of our normalization $N(\zeta ; z)$ is analytic in both arguments and $M(\zeta ; z)$ is analytic in $\zeta$ and anti-analytic in $z$.

In summary, we have

$$
\begin{aligned}
Q^{\prime}(a ; u, v) & =N(v ; a)-N(u ; a), \\
\left(P^{\prime}(a ; u, v)\right)^{+} & =M(u ; a)-M(v ; a), \\
M^{\prime}(u ; v) & =\left(M^{\prime}(v ; u)\right)^{+}, \\
N^{\prime}(u ; v) & =N^{\prime}(v ; u),
\end{aligned}
$$$$
w_{\nu}(u)-w_{\nu}(v)=\sum_{\mu=1}^{n} k_{\mu}(u, v) P_{\mu \nu},
$$$$
\nu=1,2, \cdots, n,
$$

$$
w_{\nu}^{\prime}(u)=\sum_{\mu=1}^{n} l_{\mu}(u) P_{\mu \nu}
$$$$
\nu=1,2, \cdots, n \text {, }
$$

(103) $p(v ; \zeta)-p(u ; \zeta)=Q(\zeta ; u, v)+(P(\zeta ; u, v))^{+}-\sum_{\mu=1}^{n} k_{\mu}(u, v) \omega_{\mu}(\zeta)$,

$(108) \pi(v ; \zeta)-\pi(u ; \zeta)=Q(\zeta ; u, v)-(P(\zeta ; u, v))^{+}$,

$$
Q(\zeta ; u, v)=\frac{1}{2}[\{p(v ; \zeta)-p(u ; \zeta)\}+\{\pi(v ; \zeta)-\pi(u ; \zeta)\}]
$$

$$
\begin{aligned}
& +\frac{1}{2} \sum_{\mu=1}^{n} k_{\mu}(u, v) \omega_{\mu}(\zeta), \\
(P(\zeta ; u, v))^{+}= & \frac{1}{2}[\{p(v ; \zeta)-p(u ; \zeta)\}-\{\pi(v ; \zeta)-\pi(u ; \zeta)\}] \\
& +\frac{1}{2} \sum_{\mu=1}^{n} k_{\mu}(u, v) \omega_{\mu}(\zeta), \\
N(\zeta ; z)= & \frac{1}{2}\left[\pi^{\prime}(z ; \zeta)+p^{\prime}(z ; \zeta)\right]-\frac{1}{2} \sum_{\mu=1}^{n} l_{\mu}(z) \omega_{\mu}(\zeta), \\
(M(\zeta ; z))^{+}= & \frac{1}{2}\left[\pi^{\prime}(z ; \zeta)-p^{\prime}(z ; \zeta)\right]+\frac{1}{2} \sum_{\mu=1}^{n} l_{\mu}(z) \omega_{\mu}(\zeta), \\
P(z ; u, v)= & \pi \sum_{\nu=1}^{\infty} f_{\nu}(z)\left[\left(f_{\nu}(u)\right)^{+}-\left(f_{\nu}(v)\right)^{+}\right] .
\end{aligned}
$$

5. Schottky functions and related classes. Schottky [15] was the first to consider the family $\Re$ of all functions $f(z)$ which are single-valued and meromorphic in $D$ and have real boundary values on $C$. He developed an interest- 
ing theory of conformal mapping of multiply-connected domains from the properties of this family and established by means of it the relation of this theory with the theory of closed Riemann surfaces. It is evident that functions $f(z) \in \Re$ are very useful in the method of contour integration.

In fact, let $f(z)$ be an arbitrary function of this family. We assume, for the sake of simplicity, that $f(z)$ has only simple poles. Let $z_{\nu}(\nu=1, \cdots, N)$ be the coordinates of the poles and $r_{\nu}(\nu=1,2, \cdots, N)$ the corresponding residues. Consider now the integral

$$
\frac{1}{2 \pi i} \oint_{C} P(z ; u, v) f^{\prime}(z) d z=-\sum_{\nu=1}^{N} r_{\nu} P^{\prime}\left(z_{\nu} ; u, v\right) .
$$

Using (16) and the fact that $f^{\prime}(z) d z$ is real on $C$, we obtain

$$
\begin{aligned}
\sum_{\nu=1}^{N} r_{\nu}^{+}\left(P^{\prime}\left(z_{\nu} ; u, v\right)\right)^{+} & =-\frac{1}{2 \pi i} \oint_{C} Q(z ; u, v) f^{\prime}(z) d z \\
& =\frac{1}{2 \pi i} \oint_{C} f(z) Q^{\prime}(z ; u, v) d z .
\end{aligned}
$$

By means of the residue theorem we deduce from (119)

$$
\sum_{\nu=1}^{N} r_{\nu}^{+}\left(P^{\prime}\left(z_{\nu} ; u, v\right)\right)^{+}=\sum_{\nu=1}^{N} r_{\nu} Q^{\prime}\left(z_{\nu} ; u, v\right)+f(u)-f(v) .
$$

Applying finally the formulas (61) and (63) we arrive at

$$
\begin{aligned}
f(v)-f(u)= & \sum_{\nu=1}^{N}\left[r_{\nu} N\left(v ; z_{\nu}\right)+r_{\nu}^{+} M\left(v ; z_{\nu}\right)\right] \\
& -\sum_{\nu=1}^{N}\left[r_{\nu} N\left(u ; z_{\nu}\right)+r_{\nu}^{+} M\left(u ; z_{\nu}\right)\right] .
\end{aligned}
$$

This proves the following theorem:

THEOREM V. Every function $f(z) \in R$ may be developed in the form

$$
f(z)=A+\sum_{\nu=1}^{N}\left[r_{\nu} N\left(z ; z_{\nu}\right)+r_{\nu}^{+} M\left(z ; z_{\nu}\right)\right] .
$$

We have now to establish the additional conditions in order that anlexpression (122) be in fact a Schottky function. Obviously, the right-hand side represents a single-valued meromorphic function of $z \in D$ with simple poles at the $z_{\nu}$. If $z$ lies on the boundary continuum $C_{\rho}$ we have by virtue of (57)

$$
f(z)=A+\sum_{\nu=1}^{N}\left[r_{\nu} N\left(z ; z_{\nu}\right)+r_{\nu}^{+}\left(N\left(z ; z_{\nu}\right)\right)^{+}\right]+\sum_{\nu=1}^{N} r_{\nu}^{+}\left(l_{p}\left(z_{\nu}\right)\right)^{+} \text {. }
$$


If we require now that $f(z)$ be real on each $C_{\rho}$, we obtain the conditions

$$
\operatorname{Im}\left\{A+\sum_{\nu=1}^{N} r_{\nu}^{+}\left(l_{\rho}\left(z_{\nu}\right)\right)^{+}\right\}=0, \quad \rho=1,2, \cdots, n .
$$

Multiplying the $\rho$ th equation (124) with $P_{\rho \mu}$ and summing over $\rho$ from 1 to $n$, we obtain in view of $(78)$ and $\left(87^{\prime}\right)$

$$
\operatorname{Im}\left\{\sum_{\nu=1}^{N} r_{\nu} w_{\mu}^{\prime}\left(z_{\nu}\right)\right\}=0, \quad \mu=1,2, \cdots, n .
$$

One recognizes easily that every function (122) for which (125) is fulfilled is of the family $\Re$ under proper choice of the constant $A$. The similarity of the conditions (125) and an analogous result of Abel for single-valued functions on closed Riemann surfaces is evident.

Another family of functions which is closely connected with $\Re$ may be defined as follows. $\&$ is the family of all functions $E(z)$ which are regular in $D$ and which satisfy on the boundary $C$ of $D$ the condition

$$
|E(z)|^{2}=E(z)(E(z))^{+}=1 \text {. }
$$

It is obvious that for every $E(z) \in \mathbb{E}$ the expression $E(z)+E(z)^{-1}$ is an element of $\Re$.

We obtain now for every function $E(z) \in \mathbb{E}$ a simple representation in terms of the functions $M(z ; u)$ and $N(z ; u)$ defined in $\$ 3$. We assume, for the sake of simplicity, that $E(z)$ has only simple zero-points in $D$ and that at each such point $n_{\nu}(\nu=1,2, \cdots, N)$ one has

$$
\lim _{z \rightarrow n_{\nu}}\left(z-n_{\nu}\right)^{-1} E(z)=r_{\nu}^{-1}, \quad \nu=1,2, \cdots, N \text {. }
$$

Because of (122), we have in view of (127)

$$
E(z)+E(z)^{-1}=A+\sum_{\nu=1}^{N}\left[r_{\nu} N\left(z ; n_{\nu}\right)+r_{\nu}^{+} M\left(z ; n_{\nu}\right)\right]
$$

with the additional condition, derived from (125),

$$
\operatorname{Im}\left\{\sum_{\nu=1}^{N} r_{\nu} w_{\mu}^{\prime}\left(n_{\nu}\right)\right\}=0, \quad \mu=1,2, \cdots, n .
$$

Since further the function $i\left[E(z)-E(z)^{-1}\right]$ is again of class $\Re$, we derive from (122), (127) and (125) the formulas

$$
\begin{aligned}
& E(z)-E(z)^{-1}=B-\sum_{\nu=1}^{N}\left[r_{\nu} N\left(z ; n_{\nu}\right)-r_{\nu}^{+} \dot{M}\left(z ; n_{\nu}\right)\right], \\
& \operatorname{Re}\left\{\sum_{\nu=1}^{N} r_{\nu} w_{\mu}^{\prime}\left(n_{\nu}\right)\right\}=0, \quad \mu=1,2, \cdots, n .
\end{aligned}
$$


Combining (128) and (129), we find the following result:

ThEOREM VI. Let $E(z)$ be a function of the class (5 with simple zeros at the points $n_{\nu}$ and let $r_{\nu}$ be the residues of $E(z)^{-1}$ at the corresponding poles. Then we have the representations:

$$
\begin{gathered}
E(z)=A_{1}+\sum_{v=1}^{N} r_{\nu}^{+} M\left(z ; n_{v}\right), \\
E(z)^{-1}=A_{2}+\sum_{v=1}^{N} r_{v} N\left(z ; n_{v}\right),
\end{gathered}
$$

and the zeros and residues are connected by the equations

$$
\sum_{\nu=1}^{N} r_{\nu} w_{\mu}^{\prime}\left(n_{\nu}\right)=0, \quad \mu=1,2, \cdots, n .
$$

These results might also have been proved directly without using the class $\Re$ by applying the method of contour integration and using the fact that because of (126) one has, on $C,(E(z))^{+}=E(z)^{-1}$.

Since the function $E(z)$ vanishes at all points $n_{\mu}$, we derive from (131) the $(N-1)$ equations

$$
\sum_{\nu=1}^{N} r_{\nu}^{+} M\left(n_{\mu} ; n_{\nu}\right)=\sum_{\nu=1}^{N} r_{\nu}^{+} M\left(n_{1} ; n_{\nu}\right), \quad \mu=2,3, \cdots, N .
$$

We want now to verify that if $N$ points $n_{\nu}$ and $N$ numbers $r_{\nu}$ can be found such that the $N+n-2$ equations (133) and (134) are satisfied then it is possible to determine constants $A_{1}$ and $A_{2}$ such that the function (131) is proportional to a function of class $\mathbb{E}$ and that (132) is proportional to its inverse. In fact, construct formally two functions $E(z)$ and $E_{1}(z)$ which equal the right-hand sides of (131) and (132). In view of (134), we may choose $A_{1}$ in such a way that $E(z)$ vanishes at all points $n_{\nu}$. Hence, the product $E(z) \cdot E_{1}(z)$ is regular in $D$, since the simple poles of $E_{1}(z)$ cancel against the zeros of $E(z)$. For $z \in C_{\rho}$ we have by virtue of (57)

$$
\begin{aligned}
E(z) \cdot E_{1}(z)= & {\left[A_{1}+\sum_{\nu=1}^{N} r_{\nu}^{+}\left(l_{\rho}\left(n_{\nu}\right)\right)^{+}+\sum_{\nu=1}^{N} r_{\nu}^{+}\left(N\left(z ; n_{\nu}\right)\right)^{+}\right] } \\
& \cdot\left[A_{2}+\sum_{\nu=1}^{N} r_{\nu} N\left(z ; n_{\nu}\right)\right] .
\end{aligned}
$$

Now, we deduce from the character of the matrix $\left(P_{\mu v}\right)$ and (133) and (78)

$$
\sum_{\nu=1}^{N} r_{\nu}^{+}\left(l_{\rho}\left(n_{\nu}\right)\right)^{+}=\sum_{\nu=1}^{N} r_{\nu}^{+}\left(l_{1}\left(n_{\nu}\right)\right)^{+}, \quad \rho=2,3, \cdots, n .
$$

If we choose now 


$$
A_{2}=A_{1}^{+}+\sum_{\nu=1}^{N} r_{\nu} l_{1}\left(n_{v}\right)
$$

we have non-negative boundary values for $E(z) E_{1}(z)$ on $C$. But since the only regular functions $f(z) \in \Re$ are real constants, we find $E(z) \cdot E_{1}(z)=k$, where $k$ is a positive constant. This proves our assertion; we may always multiply the $r_{\nu}$ 's by a common factor so that $E(z)$ becomes of class $E$.

If we introduce into the identity $E(z) \cdot E(z)^{-1}=1$ the expressions (131) and (132) and if we compute the product at a point $n_{\mu}$, we find easily

$$
r_{\mu} \sum_{\nu=1}^{N} r_{\nu}^{+} M^{\prime}\left(n_{\mu} ; n_{\nu}\right)=1, \quad \mu=1,2, \cdots, N .
$$

This system of equations for the $r_{\nu}$ 's must necessarily be a consequence of (133) and (134). Numerous other identities of similar form may be easily obtained. We shall restrict ourselves here to one example.

We may eliminate the constant $A_{1}$ in (131) by using the fact that $E\left(n_{1}\right)$ $=0$, and this gives to (131) the form

$$
E(z)=\sum_{\nu=1}^{N} r_{\nu}^{+}\left[M\left(z ; n_{\nu}\right)-M\left(n_{1} ; n_{\nu}\right)\right],
$$

which is in view of (63) equivalent to

$$
E(z)=\sum_{\nu=1}^{N} r_{\nu}^{+}\left(P^{\prime}\left(n_{\nu} ; z, n_{1}\right)\right)^{+}
$$

This is another standard representation for the class $\&$ in which the constant term has been determined. Let now

$$
F(z)=\sum_{\mu=1}^{M} s_{\mu}^{+}\left(P^{\prime}\left(m_{\mu} ; z, m_{1}\right)\right)^{+}
$$

be another function of class $(₹$. For the sake of simplicity, we assume that no zero of $E(z)$ coincides with one of $F(z)$. The function $E(z) \cdot F(z)$ is again of the same class $₹$. Hence, multiplying on the one hand (131') with (137) and using, on the other hand, the representation $\left(131^{\prime \prime}\right)$ for the product, we obtain

$$
\begin{aligned}
\sum_{\mu=1}^{M} & \sum_{\nu=1}^{N} r_{\nu}^{+} s_{\mu}^{+}\left(P^{\prime}\left(n_{\nu} ; z, n_{1}\right)\right)^{+}\left(P^{\prime}\left(m_{\mu} ; z, m_{1}\right)\right)^{+} \\
& =\sum_{\nu=1}^{N} r_{\nu}^{+}\left(F\left(n_{\nu}\right)^{-1}\right)^{+}\left(P^{\prime}\left(n_{\nu} ; z, n_{1}\right)\right)^{+}+\sum_{\mu=1}^{M} s_{\mu}^{+}\left(E\left(m_{\mu}\right)^{-1}\right)^{+}\left(P^{\prime}\left(m_{\mu} ; z, n_{1}\right)\right)^{+},
\end{aligned}
$$

an interesting relation between the left-hand second order expressions of $P^{\prime}$ and the linear terms on the right-hand side. Using the above procedure with respect to the product $E(z)^{-1} \cdot F(z)^{-1}$ gives an analogous identity in $Q^{\prime}$. 
We consider now the particular function (43) of class $\mathbb{E}$ and apply to it the representation formula $\left(131^{\prime \prime}\right)$. We obtain

$$
P^{\prime}(z ; u, v) Q^{\prime}(z ; u, v)^{-1}=\sum_{\nu=1}^{2 n} r_{\nu}^{+}\left(P^{\prime}\left(n_{\nu} ; z, u\right)\right)^{+},
$$

where the summation is extended over all $2 n$ zeros $n_{\nu}$ of the left-hand expression and it is to be noted that $z=u$ and $z=v$ are zeros of this function. This formula shows that $Q^{\prime}(z ; u, v)$ may be expressed by means of $P^{\prime}(z ; u, v)$ and $P^{\prime}\left(n_{\nu} ; z, u\right)$. This result is of interest because $P(z ; u, v)$ is a kernel function and, therefore, numerically easy to handle. The determination of the $n_{v}$ and $r$, will, however, be in general so difficult that little immediate use of (139) is to be expected.

Let us consider, further, the matrix $\left(M^{\prime}\left(z_{\nu} ; z_{\mu}\right)\right)$ which occurred in the equation (136) for the residues $r_{\nu}$. Because of (65) this matrix is Hermitian. In order to study it in more detail, we remark that for every function $f(z)$ which is single-valued and continuously differentiable in $D+C$ we have because of (57)

$$
\begin{aligned}
\left(f(z),(M(z ; u))^{+}\right) & =\frac{1}{2 i} \oint_{C} f^{\prime}(z)(M(z ; u))^{+} d z \\
& =\frac{1}{2 i} \oint_{C} f^{\prime}(z) N(z ; u) d z=\pi f^{\prime}(u) .
\end{aligned}
$$

By the usual reasoning we conclude for every function $f(z) \in \Omega$

$$
\left(f(z),(M(z ; u))^{+}\right)=\pi f^{\prime}(u) .
$$

In particular, we derive from $\left(140^{\prime}\right)$ for every function of the type

$$
f(z)=A+\sum_{\nu=1}^{N} \lambda_{\nu} M\left(z ; n_{\nu}\right)
$$

which is obviously of the class $\Omega$, the relation

$$
\left(f, f^{+}\right)=\iint_{D}\left|f^{\prime}(z)\right|^{2} d x d y=\pi \sum_{\mu, \nu=1}^{N} \lambda_{\nu} \lambda_{\mu}^{+} M^{\prime}\left(n_{\nu} ; n_{\mu}\right) .
$$

This shows that the Hermitian form with matrix $\left(M^{\prime}\left(n_{\nu} ; n_{\mu}\right)\right)$ is positive-definite. If we choose in particular as $f(z)$ a function $E(z) \in \mathbb{E}$, we find

$$
\left(E, E^{+}\right)=\iint_{D}\left|E^{\prime}(z)\right|^{2} d x d y=\sum_{\mu, \nu=1}^{N} r_{\nu} r_{\mu}^{+} M^{\prime}\left(n_{\nu} ; n_{\mu}\right) \cdot \pi .
$$

We find from (136) that the value of this sum is $N \cdot \pi$. This result is easily understood; each function $E(z)$ maps $D$ upon the unit circle covered $N$ times and the area of this image domain is exactly $N \cdot \pi$. 
There is another interesting representation for all functions $f(z) \in \Re$. Let

$$
w^{\prime}(z)=\sum_{\nu=1}^{n} \xi_{\nu} w_{\nu}^{\prime}(z)
$$

$\xi_{\nu}$ real,

be an arbitrary linear combination of derivatives of harmonic measures with real coefficients $\xi_{\nu}$. If $f(z) \in \Re$ with $N$ simple poles $z_{\nu}$ and corresponding residues $r_{\nu}$ is given, consider the function $f(z) w^{\prime}(z)$. We have, evidently, on $C$

$$
\left(f(z) w^{\prime}(z) d z\right)^{+}=-f(z) w^{\prime}(z) d z,
$$

$z$ varies on $C$.

Consider now the equation

$$
\frac{1}{2 \pi i} \oint_{C} M(z ; u) f(z) w^{\prime}(z) d z=\sum_{\nu=1}^{N} r_{\nu} w^{\prime}\left(z_{\nu}\right) M\left(z_{\nu} ; u\right) .
$$

Because of (57) and (144), we obtain

$$
\sum_{\nu=1}^{N}\left(r_{\nu} w^{\prime}\left(z_{\nu}\right) M\left(z_{\nu} ; u\right)\right)^{+}=\frac{1}{2 \pi i} \sum_{\mu=1}^{n} \oint_{c_{\mu}}\left[N(z ; u)+l_{\mu}(u)\right] f(z) w^{\prime}(z) d z,
$$

whence by means of the residue theorem

$$
\begin{aligned}
f(u) w^{\prime}(u)= & -\sum_{\nu=1}^{N} r_{\nu} w^{\prime}\left(z_{\nu}\right) N\left(z_{\nu} ; u\right)+\sum_{\nu=1}^{N} r_{\nu}^{+}\left(w^{\prime}\left(z_{\nu}\right) M\left(z_{\nu} ; u\right)\right)^{+} \\
& -\sum_{\mu=1}^{n} l_{\mu}(u) a_{\mu}
\end{aligned}
$$

where

$$
a_{\mu}=\frac{1}{2 \pi i} \oint_{c_{\mu}} f(z) w^{\prime}(z) d z, \quad \quad \mu=1,2, \cdots, n,
$$

are, because of (144), real constants. Considering, finally, (78) we find:

THEOREM VII. Every function $f(z) \in \Re$ may be represented in the form

$$
f(z) w^{\prime}(z)=\sum_{\nu=1}^{N}\left[r_{\nu}^{+}\left(w^{\prime}\left(z_{\nu}\right)\right)^{+}\left(M\left(z_{\nu} ; z\right)\right)^{+}-r_{\nu} w^{\prime}\left(z_{\nu}\right) N\left(z_{\nu} ; z\right)\right]+\sum_{\mu=1}^{n} \lambda_{\mu} w_{\mu}^{\prime}(z),
$$

with real coefficients $\lambda_{\mu}$.

We make the following application of this result:

Take an arbitrary function $w^{\prime}(z)$ of the type (143); then we have in

$$
f(z)=w_{i}^{\prime}(z) / w^{\prime}(z)
$$

a function of the class $\Re$. Suppose that all zeros $z_{\nu}$ of $w^{\prime}(z)$ are simple; it follows easily from the argument principle that there are exactly $n-2$ of them in 
$D$. We assume in the following considerations $n>2$ so that the existence of zeros $z_{\nu}$ is assured. The corresponding residues of $f(z)$ are

$$
r_{\nu}=w_{i}^{\prime}\left(z_{v}\right) / w^{\prime \prime}\left(z_{v}\right) \text {. }
$$

We apply now formula (147) with the function $w^{\prime}(z)$ replaced there by $w_{k}^{\prime}(z)$; we have

$$
\begin{aligned}
\frac{w_{i}^{\prime}(z) w_{k}^{\prime}(z)}{w^{\prime}(z)}-\sum_{\mu=1}^{n} \lambda_{i k, \mu} w_{\mu}^{\prime}(z)= & \sum_{\nu=1}^{n-2}\left(\left[\frac{w_{i}^{\prime}\left(z_{\nu}\right) w_{k}^{\prime}\left(z_{\nu}\right)}{w^{\prime \prime}\left(z_{\nu}\right)}\right]\right)^{+}\left(M\left(z_{\nu} ; z\right)\right)^{+} \\
& -\sum_{\nu=1}^{n-2} \frac{w_{i}^{\prime}\left(z_{\nu}\right) w_{k}^{\prime}\left(z_{\nu}\right)}{w^{\prime \prime}\left(z_{\nu}\right)} N\left(z_{\nu} ; z\right) .
\end{aligned}
$$

Since there are $n-1$ linearly independent $w_{i}^{\prime}(z)$, we may write down $n(n-1) / 2$ equations (149) for a given denominator $w^{\prime}(z)$. We may consider the $2 n-4$ terms $M\left(z_{\nu} ; z\right)$ and $N\left(z_{\nu} ; z\right)$ as unknowns and the equations (149) as a system for their determination. The number of linearly independent equations (149) is of great importance from this point of view.

First we conclude from (149) that there are at most $3 n-6$ linearly independent terms $w_{i}^{\prime}(z) w_{k}^{\prime}(z)$. In fact, choose $w^{\prime}(z)$ in (149) as $w_{3}^{\prime}(z)$. Take all equations (149) with $w_{1}^{\prime}(z) w_{k}^{\prime}(z) / w_{3}^{\prime}(z)$ and $w_{2}^{\prime}(z) w_{k}^{\prime}(z) / w_{3}^{\prime}(z)(k=1,2,4$, $\cdots, n-1)$. These are exactly $2 n-5$ equations. If now another equation (149) of the form $w_{i}^{\prime}(z) w_{k}^{\prime}(z) / w_{3}^{\prime}(z)$ is considered with $i, k \neq 1,2,3$, we have $2 n-4$ equations with $2 n-4$ unknowns. Further, applying condition (125) to our functions $f(z)$ we have in each case

$$
\sum_{v=1}^{n-2}\left(\left[\frac{w_{i}^{\prime}\left(z_{\nu}\right) w_{k}^{\prime}\left(z_{\nu}\right)}{w^{\prime \prime}\left(z_{\nu}\right)}\right]\right)^{+}-\sum_{\nu=1}^{n-2} \frac{w_{i}^{\prime}\left(z_{\nu}\right) w_{k}^{\prime}\left(z_{v}\right)}{w^{\prime \prime}\left(z_{v}\right)}=0 .
$$

This shows that the rank of the matrix of our $2 n-4$ equations is at most $2 n-5$. There exist, therefore, constants such that

$$
\begin{aligned}
\alpha w_{i}^{\prime}(z) w_{k}^{\prime}(z)+\sum_{\nu=1}^{n-1} \beta_{\nu} w_{1}^{\prime}(z) w_{\nu}^{\prime}(z)+\sum_{\nu=1}^{n-1} \gamma_{\nu} w_{2}^{\prime}(z) w_{\nu}^{\prime}(z) & \\
& +\sum_{\nu=1}^{n-1} \delta_{\nu} w_{3}^{\prime}(z) w_{\nu}^{\prime}(z)=0 .
\end{aligned}
$$

In the same fashion we show easily that each set of $3 n-5$ terms $w_{i}^{\prime}(z) w_{k}^{\prime}(z)$ is linearly dependent and that all terms may be obtained as linear combinations of at most $3 n-6$ basic independent products of this form.

Theorem VIII. Among the expressions $w_{i}^{\prime}(z) w_{k}^{\prime}(z)$ there are at most $3 n-6$ linearly independent ones.

Let us assume now that we can find $2 n-5$ combinations of indices $i$ and $k$ for which the equations (149) are linearly independent. This assumption is 
in general fulfilled, as follows from a theorem of Noether (cf. [8, pp. 502527]) in the theory of algebraic integrals. If we want to solve the system of equations, we have first to take notice of the relations (150) between the coefficients of this system. Since by virtue of the residue theorem

$$
\sum_{i=1}^{n-2} \frac{w_{i}^{\prime}\left(z_{v}\right) w_{k}^{\prime}\left(z_{v}\right)}{w^{\prime \prime}\left(z_{v}\right)}=\frac{1}{2 \pi i} \oint_{c} \frac{w_{i}^{\prime}(z) w_{k}^{\prime}(z)}{w^{\prime}(z)} d z,
$$

we may put the equations (149) in the form

$$
\begin{aligned}
\frac{w_{i}^{\prime}(z) w_{k}^{\prime}(z)}{w^{\prime}(z)} & -\sum_{\mu=1}^{n} \lambda_{i k, \mu} w_{\mu}^{\prime}(z) \\
= & \sum_{\nu=2}^{n-2}\left(\left[\frac{w_{i}^{\prime}\left(z_{v}\right) w_{k}^{\prime}\left(z_{v}\right)}{w^{\prime \prime}\left(z_{\nu}\right)}\right]\right)^{+}\left(\left(M\left(z_{\nu} ; z\right)\right)^{+}-\left(M\left(z_{1} ; z\right)\right)^{+}\right) \\
& -\sum_{\nu=2}^{n-2} \frac{w_{i}^{\prime}\left(z_{\nu}\right) w_{k}^{\prime}\left(z_{\nu}\right)}{w^{\prime \prime}\left(z_{\nu}\right)}\left(N\left(z_{\nu} ; z\right)-N\left(z_{1} ; z\right)\right) \\
& -\left(N\left(z_{1} ; z\right)-\left(M\left(z_{1} ; z\right)\right)^{+}\right) \cdot \frac{1}{2 \pi i} \oint_{c} \frac{w_{i}^{\prime} w_{k}^{\prime}}{w^{\prime}} d z .
\end{aligned}
$$

Applying finally $\left(103^{\prime}\right)$ we find

$$
\begin{aligned}
\frac{w_{i}^{\prime}(z) w_{k}^{\prime}(z)}{w^{\prime}(z)} & -\sum_{\mu=1}^{n} \lambda_{i k, \mu} w_{\mu}^{\prime}(z)-\sum_{\mu=1}^{n} l_{\mu}(z) \omega_{\mu}\left(z_{1}\right) \cdot \frac{1}{2 \pi i} \oint_{c} \frac{w_{i} w_{k}^{\prime}}{w^{\prime}} d z \\
= & \sum_{\nu=2}^{n-2}\left(\left[\frac{w_{i}^{\prime}\left(z_{v}\right) w_{k}^{\prime}\left(z_{v}\right)}{w^{\prime \prime}\left(z_{\nu}\right)}\right]\right)^{+}\left(\left(M\left(z_{\nu} ; z\right)\right)^{+}-\left(M\left(z_{1} ; z\right)\right)^{+}\right) \\
& -\sum_{\nu=2}^{n-2} \frac{w_{i}^{\prime}\left(z_{v}\right) w_{k}^{\prime}\left(z_{v}\right)}{w^{\prime \prime}\left(z_{v}\right)}\left(N\left(z_{v} ; z\right)-N\left(z_{1} ; z\right)\right) \\
& -\frac{p^{\prime}\left(z ; z_{1}\right)}{2 \pi i} \oint_{c} \frac{w_{i}^{\prime} w_{k}^{\prime}}{w^{\prime}} d z .
\end{aligned}
$$

From this system of equations in $2 n-5$ unknowns with nonvanishing determinant we may compute the unknowns. We find:

Theorem IX. The functions $p^{\prime}\left(z ; z_{1}\right),\left\{N\left(z_{p} ; z\right)-N\left(z_{1} ; z\right)\right\}$ and $\left\{\left(M\left(z_{p} ; z\right)\right)^{+}\right.$ $\left.-\left(M\left(z_{1} ; z\right)\right)^{+}\right\}$are rational functions of the functions $w_{i}^{\prime}(z)(i=1, \cdots$, $n-1)$ with coefficients depending on $z_{1}$.

For example, we have

$$
p^{\prime}\left(z ; z_{1}\right)=\frac{\sum a_{i k}\left(z_{1}\right) w_{i}^{\prime}(z) w_{k}^{\prime}(z)}{\sum_{v=1}^{n} \xi_{\nu} w_{\nu}^{\prime}(z)},
$$


where the summation in the numerator is to be extended over any basic system of the $w_{i}^{\prime}(z) w_{k}^{\prime}(z)$. The coefficients $\xi_{\nu}$ in the denominator depend also on $z_{1}$, since the denominator has to vanish at this point. We might choose as such a denominator the expression

$$
w^{\prime}(z)=\frac{1}{i}\left|\begin{array}{ccc}
w_{1}^{\prime}(z) & w_{2}^{\prime}(z) & w_{3}^{\prime}(z) \\
w_{1}^{\prime}\left(z_{1}\right) & w_{2}^{\prime}\left(z_{1}\right) & w_{3}^{\prime}\left(z_{1}\right) \\
\left(w_{1}^{\prime}\left(z_{1}\right)\right)^{+} & \left(w_{2}^{\prime}\left(z_{1}\right)\right)^{+} & \left(w_{3}^{\prime}\left(z_{1}\right)\right)^{+}
\end{array}\right|,
$$

if it is not identically zero, which occurs only at exceptional points $z_{1}$.

It is possible to obtain additional information about the representation $\left(153^{\prime}\right)$ in the following way. Introduce the differential operators

$$
\begin{aligned}
\frac{\partial}{\partial z} & =\frac{1}{2}\left(\frac{\partial}{\partial x}-i \frac{\partial}{\partial y}\right), & \frac{\partial}{\partial z_{1}} & =\frac{1}{2}\left(\frac{\partial}{\partial x_{1}}-i \frac{\partial}{\partial y_{1}}\right), \\
z & =x+i y, & z_{1} & =x_{1}+i y_{1} .
\end{aligned}
$$

Differentiating $\left(153^{\prime}\right)$ with respect to $z_{1}$ and using the definition of $p^{\prime}\left(z ; z_{1}\right)$, we find

$$
\frac{\partial^{2} g\left(z ; z_{1}\right)}{\partial z \partial z_{1}}=\frac{\sum A_{\kappa \lambda \mu}\left(z_{1}\right) w_{\kappa}^{\prime}(z) w_{\lambda}^{\prime}(z) w_{\mu}^{\prime}(z)}{\left[\sum_{\nu=1}^{n} \xi_{\nu}\left(z_{1}\right) w_{\nu}^{\prime}(z)\right]^{2}} .
$$

But this expression is symmetric with respect to $z$ and $z_{1}$; hence we have here a rational expression in $w_{\nu}^{\prime}(z)$ and $w_{\nu}^{\prime}\left(z_{1}\right)$ for a second derivative of Green's function. We do not pursue these formal considerations; they show the importance of the method of contour integration for studying identities between domain functions. It appears that for the general domain $D$ of connectivity $n>2$ the derivatives of the harmonic measures are among the most important domain functions, since many others may be constructed rationally from them. The last results of this section are closely related to Noether's theorem [8] on algebraic integrals. In view of Schottky's theorem on the mapping of multiply-connected domains on Riemann surfaces they might even be derived directly from Noether's result. Our method leads to them directly by simple applications of Cauchy's theorem and seems to be more appropriate for the theory of conformal mapping.

6. Theory of variation for the fundamental domain functions. In this chapter the method of contour integration will be applied in order to study the variation of domain functions under an infinitesimal change of the domain $D$. We shall vary the domain $D$ as follows; we choose a fixed point $z_{0} \in D$ and consider the conformal mapping

$$
z^{*}=z+\frac{e^{i \phi} \rho^{2}}{z-z_{0}}, \quad 0 \leqq \phi<2 \pi, 0<\rho .
$$


This map is univalent in the domain $\left|z-z_{0}\right|>\rho$. If we choose $\rho$ sufficiently small, the whole boundary $C$ of $D$ will lie in this domain of univalency and will, therefore, be mapped by (157) upon a set of $n$ smooth curves $C^{*}$. These curves bound a new domain $D^{*}$ which will be less different from $D$ the smaller $\rho$ is chosen. It is this domain $D^{*}$ for which we want to compute the fundamental domain functions in terms of those belonging to $D$. We shall denote the domain functions of $D^{*}$ by the same letters as those of $D$, but shall indicate their new domain by an asterisk. Thus $P^{*}(z ; u, v)$ has with respect to $D^{*}$ the same meaning as $P(z ; u, v)$ has with respect to $D$.

In applying the method of contour integration, we shall have to eliminate from $D$ a small circle of radius $\epsilon$ around $z_{0}$. We denote the circumference by $\gamma_{\epsilon}$ and we denote the remaining part of $D$ by $D_{\epsilon}$. By virtue of Cauchy's theorem, we have

$$
\begin{aligned}
\frac{1}{2 \pi i} \oint_{c} P^{*}\left(z^{*}(z) ; u^{*}, v^{*}\right) P^{\prime}(z ; a, b) d z & \\
& =\frac{1}{2 \pi i} \oint_{\gamma_{\epsilon}} P^{*}\left(z^{*}(z) ; u^{*}, v^{*}\right) P^{\prime}(z ; a, b) d z
\end{aligned}
$$

since $z^{*}(z)$ is a regular analytic function of $z$ in $D_{\epsilon}$. On $\gamma_{\epsilon}$ we may develop $P^{*}\left(z^{*} ; u^{*}, v^{*}\right)$ into a series in powers of $\rho^{2} / \epsilon$ which will converge if $\epsilon \gg \rho^{2}$.

We find, in fact,

$$
\begin{aligned}
& P^{*}\left(z^{*} ; u^{*}, v^{*}\right)=P^{*}\left(z ; u^{*}, v^{*}\right)+\frac{e^{i \phi} \rho^{2}}{z-z_{0}} P^{* \prime}\left(z ; u^{*}, v^{*}\right)+\cdots ; \\
& P^{* \prime}\left(z ; u^{*}, v^{*}\right)=\frac{d}{d z} P^{*}\left(z ; u^{*}, v^{*}\right) .
\end{aligned}
$$

Introducing this development into the right-hand integral of (158), remarking that $P^{*}\left(z ; u^{*}, v^{*}\right)$ is regular in the interior of $\gamma_{\epsilon}$ and applying the residue theorem, we obtain

$$
\begin{aligned}
\frac{1}{2 \pi i} \oint_{\gamma_{\epsilon}} P^{*}\left(z^{*}(z) ; u^{*}, v^{*}\right) P^{\prime}(z ; a, b) d z & \\
& =e^{i \phi} \rho^{2} P^{* \prime}\left(z_{0} ; u^{*}, v^{*}\right) P^{\prime}\left(z_{0} ; a, b\right)+o\left(\rho^{2}\right)
\end{aligned}
$$

where $o\left(\rho^{2}\right)$ shall henceforth always denote a corrective term of higher order in $\rho$ such that $\lim _{\rho \rightarrow 0} \rho^{-2} o\left(\rho^{2}\right)=0$. It is obvious that (159) permits the computation of the higher terms in $\rho$, too; the remainder term may be estimated in terms of the values of $P^{*}\left(z ; u^{*}, v^{*}\right)$ on $\gamma_{\epsilon}$.

We apply now formula $(16)$ for $P(z ; a, b)$ and the corresponding relation for $P^{*}\left(z^{*} ; u^{*}, v^{*}\right)$; in fact, if $z$ lies on $C$, the varied point $z^{*}$ will lie on $C^{*}$, so that both instances of (16) hold simultaneously. We find: 


$$
\begin{aligned}
\frac{1}{2 \pi i} \sum_{\mu=1}^{n} \oint_{c_{\mu}}\left[Q^{*}\left(z^{*}(z) ; u^{*}, v^{*}\right)-k_{\mu}^{*}\left(u^{*}, v^{*}\right)\right] Q^{\prime}(z ; a, b) d z \\
=-e^{-i \phi} \rho^{2}\left(P^{* \prime}\left(z_{0} ; u^{*}, v^{*}\right)\right)^{+}\left(P^{\prime}\left(z_{0} ; a, b\right)\right)^{+}+o\left(\rho^{2}\right)
\end{aligned}
$$

On the other hand, we obtain from the residue theorem, applied to $D_{\epsilon}$,

$$
\begin{aligned}
\frac{1}{2 \pi i} \oint_{c} Q^{*}\left(z^{*} ; u^{*}, v^{*}\right) Q^{\prime}(z ; a, b) d z & =Q^{*}\left(a^{*} ; u^{*}, v^{*}\right)-Q^{*}\left(b^{*} ; u^{*}, v^{*}\right)-Q(u ; a, b)+Q(v ; a, b) \\
& +\frac{1}{2 \pi i} \oint_{\gamma_{\epsilon}} Q^{*}\left(z^{*} ; u^{*}, v^{*}\right) Q^{\prime}(z ; a, b) d z .
\end{aligned}
$$

From a development of $Q^{*}\left(z^{*} ; u^{*}, v^{*}\right)$ on $\gamma_{\epsilon}$ such as we used in (159) for $P^{*}\left(z^{*} ; u^{*}, v^{*}\right)$ we get

$$
\begin{aligned}
\frac{1}{2 \pi i} \oint_{\gamma_{\epsilon}} Q^{*}\left(z^{*} ; u^{*}, v^{*}\right) Q^{\prime}(z ; a, b) & d z \\
& =e^{i \phi} \rho^{2} Q^{* \prime}\left(z_{0} ; u^{*}, v^{*}\right) Q^{\prime}\left(z_{0} ; a, b\right)+o\left(\rho^{2}\right)
\end{aligned}
$$

In view of the uniform convergence of $Q^{*}\left(z ; u^{*}, v^{*}\right)$ and $P^{*}\left(z ; u^{*}, v^{*}\right)$ to the functions $Q(z ; u, v), P(z ; u, v)$ in each closed subdomain of $D$ with $\rho \rightarrow 0$ and because of the symmetry rule (49), we obtain from (161), (162) and (163) the variation formula

$$
\begin{aligned}
& Q^{*}\left(u^{*} ; a^{*}, b^{*}\right)-Q^{*}\left(v^{*} ; a^{*}, b^{*}\right)=Q(u ; a, b)-Q(v ; a, b) \\
& -e^{i \phi} \rho^{2} Q^{\prime}\left(z_{0} ; u, v\right) Q^{\prime}\left(z_{0} ; a, b\right)-e^{-i \phi} \rho^{2}\left(P^{\prime}\left(z_{0} ; u, v\right)\right)^{+}\left(P^{\prime}\left(z_{0} ; a, b\right)\right)^{+}+o\left(\rho^{2}\right) .
\end{aligned}
$$

This formula expresses the variation of the symmetric expression $Q(u ; a, b)$ $-Q(v ; a, b)$ in terms of the unvaried domain functions $P$ and $Q$. One sees how the method of contour integration leads immediately to this important result.

Just in the same way, we obtain from the identities

$$
\begin{aligned}
& \frac{1}{2 \pi i} \oint_{c} Q^{*}\left(z^{*} ; u^{*}, v^{*}\right) P^{\prime}(z ; a, b) d z \\
& \quad=P(v ; a, b)-P(u ; a, b)+\frac{1}{2 \pi i} \oint_{\gamma_{\epsilon}} Q^{*}\left(z^{*} ; u^{*}, v^{*}\right) P^{\prime}(z ; a, b) d z
\end{aligned}
$$

and

$$
\begin{aligned}
& \frac{1}{2 \pi i} \oint_{C} P^{*}\left(z^{*} ; u^{*}, v^{*}\right) Q^{\prime}(z ; a, b) d z \\
& =P^{*}\left(a^{*} ; u^{*}, v^{*}\right)-P^{*}\left(b^{*} ; u^{*}, v^{*}\right)+\frac{1}{2 \pi i} \oint_{\gamma_{\epsilon}} P^{*}\left(z^{*} ; u^{*}, v^{*}\right) Q^{\prime}(z ; a, b) d z,
\end{aligned}
$$


by use of the relations (16) on $C$ the final result

$$
\begin{aligned}
& P^{*}\left(u^{*} ; a^{*}, b^{*}\right)-P^{*}\left(v^{*} ; a^{*}, b^{*}\right)=P(u ; a, b)-P(v ; a, b) \\
& \quad-e^{i \phi} \rho^{2} Q^{\prime}\left(z_{0} ; u, v\right) P^{\prime}\left(z_{0} ; a, b\right)-e^{-i \phi} \rho^{2}\left(P^{\prime}\left(z_{0} ; u, v\right) Q^{\prime}\left(z_{0} ; u, v\right)\right)^{+}+o\left(\rho^{2}\right) .
\end{aligned}
$$

Next we transform, by similar methods, the two identities

$$
\begin{aligned}
& \frac{1}{2 \pi i} \oint_{C} P^{*}\left(z^{*} ; u^{*}, v^{*}\right) w_{\nu}^{\prime}(z) d z=\frac{1}{2 \pi i} \oint_{\gamma_{\epsilon}} P^{*}\left(z^{*} ; u^{*}, v^{*}\right) w_{\nu}^{\prime}(z) d z \\
& \frac{1}{2 \pi i} \sum_{\mu=1}^{n} \oint_{C_{\mu}}\left[Q^{*}\left(z^{*} ; u^{*}, v^{*}\right)-k_{\mu}^{*}\left(u^{*}, v^{*}\right)\right] w_{\nu}^{\prime}(z) d z \\
& =w_{\nu}(v)-w_{\nu}(u)+\sum_{\mu=1}^{n} k_{\mu}^{*}\left(u^{*}, v^{*}\right) P_{\mu \nu}+\frac{1}{2 \pi i} \oint_{\gamma_{\epsilon}} Q^{*}\left(z^{*} ; u^{*}, v^{*}\right) w_{\nu}^{\prime}(z) d z
\end{aligned}
$$

Using series developments on $\gamma_{\epsilon}$ and the formulas (16) and (71), we obtain

$$
\begin{aligned}
\sum_{\mu=1}^{n} k_{\mu}^{*}\left(u^{*}, v^{*}\right) P_{\mu \nu}= & w_{\nu}(u)-w_{\nu}(v)-e^{i \phi} \rho^{2} Q^{\prime}\left(z_{0} ; u, v\right) w_{\nu}^{\prime}\left(z_{0}\right) \\
& -e^{-i \phi} \rho^{2}\left(P^{\prime}\left(z_{0} ; u, v\right)\right)^{+}\left(w_{\nu}^{\prime}\left(z_{0}\right)\right)^{+}+o\left(\rho^{2}\right) .
\end{aligned}
$$

Because of (75) this yields

$$
\begin{aligned}
\sum_{\mu=1}^{n}\left[k_{\mu}^{*}\left(u^{*}, v^{*}\right)-k_{\mu}(u, v)\right] P_{\mu \nu} & =-e^{i \phi} \rho^{2} Q^{\prime}\left(z_{0} ; u, v\right) w_{\nu}^{\prime}\left(z_{0}\right) \\
& -e^{-i \phi} \rho^{2}\left(P^{\prime}\left(z_{0} ; u, v\right)\right)^{+}\left(w_{\nu}^{\prime}\left(z_{0}\right)\right)^{+}+o\left(\rho^{2}\right) .
\end{aligned}
$$

In view of the equations $(78)$ and the character of the matrix $\left(P_{\mu \nu}\right)$ this leads to

$$
\begin{aligned}
k_{\mu}^{*}\left(u^{*}, v^{*}\right)= & k_{\mu}(u, v)-e^{i \phi} \rho^{2} Q^{\prime}\left(z_{0} ; u, v\right) l_{\mu}\left(z_{0}\right) \\
& -e^{-i \phi} \rho^{2}\left(P^{\prime}\left(z_{0} ; u, v\right)\right)^{+}\left(l_{\mu}\left(z_{0}\right)\right)^{+}+A\left(z_{0} ; u, v\right)+o\left(\rho^{2}\right),
\end{aligned}
$$

where the function $A\left(z_{0} ; u, v\right)$ does not depend on the index $\mu$.

The $k_{\mu}(u, v)$ are by virtue of $(75)$ closely related to the harmonic measures $\omega_{\nu}(z)$. The variation formulas for $\omega_{\nu}(z)$ and its periods $P_{\mu \nu}$ under variations (157) are well known. We have [12]

$$
\begin{aligned}
\omega_{\nu}^{*}\left(z^{*}\right)=\omega_{\nu}(z)+2^{-1} e^{i \phi} \rho^{2} p^{\prime}\left(z_{0} ; z\right) w_{\nu}^{\prime}\left(z_{0}\right) \\
+2^{-1} e^{-i \phi} \rho^{2}\left(p^{\prime}\left(z_{0} ; z\right)\right)^{+}\left(w_{\nu}^{\prime}\left(z_{0}\right)\right)^{+}+o\left(\rho^{2}\right)
\end{aligned}
$$

and

$$
\begin{aligned}
P_{\mu \nu}^{*}= & P_{\mu \nu}+2^{-1} e^{i \phi} \rho^{2} w_{\nu}^{\prime}\left(z_{0}\right) w_{\mu}^{\prime}\left(z_{0}\right) \\
& +2^{-1} e^{-i \phi} \rho^{2}\left(w_{\nu}^{\prime}\left(z_{0}\right)\right)^{+}\left(w_{\mu}^{\prime}\left(z_{0}\right)\right)^{+}+o\left(\rho^{2}\right) .
\end{aligned}
$$


These formulas could also have been derived by our method of contour integration, but we omit this proof for the sake of brevity. They are very useful in the following considerations. We start with the two obvious equations

$$
\begin{aligned}
& \frac{1}{2 \pi i} \oint_{c} P^{*}\left(z^{*} ; u^{*}, v^{*}\right) d p(z ; a)=-P^{*}\left(a^{*} ; u^{*}, v^{*}\right) \\
&+e^{i \phi} \rho^{2} P^{\prime}\left(z_{0} ; u, v\right) p^{\prime}\left(z_{0} ; a\right)+o\left(\rho^{2}\right) \\
& \frac{1}{2 \pi i} \sum_{\mu=1}^{n} \oint_{c_{\mu}}\left[Q^{*}\left(z^{*} ; u^{*}, v^{*}\right)-k_{\mu}^{*}\left(u^{*}, v^{*}\right)\right] d p(z ; a) \\
&=-Q^{*}\left(a^{*} ; u^{*}, v^{*}\right)+p(v ; a)-p(u ; a) \\
&+\sum_{\mu=1}^{n} k_{\mu}^{*}\left(u^{*}, v^{*}\right) \omega_{\mu}(a)+e^{i \phi} \rho^{2} Q^{\prime}\left(z_{0} ; u, v\right) p^{\prime}\left(z_{0} ; a\right)+o\left(\rho^{2}\right)
\end{aligned}
$$

By virtue of the formulas (16) we obtain from these equations

$$
\begin{aligned}
& Q^{*}\left(a^{*} ; u^{*}, v^{*}\right)+\left(P^{*}\left(a^{*} ; u^{*}, v^{*}\right)\right)^{+} \\
& =p(v ; a)-p(u ; a)+\sum_{\mu=1}^{n} k_{\mu}^{*}\left(u^{*}, v^{*}\right) \omega_{\mu}(a) \\
& \quad+e^{i \phi} \rho^{2} Q^{\prime}\left(z_{0} ; u, v\right) p^{\prime}\left(z_{0} ; a\right)+e^{-i \phi} \rho^{2}\left(P^{\prime}\left(z_{0} ; u, v\right)\right)^{+}\left(p^{\prime}\left(z_{0} ; a\right)\right)^{+}+o\left(\rho^{2}\right) .
\end{aligned}
$$

Applying now the formula (103), we find

$$
\begin{aligned}
& p^{*}\left(v^{*} ; a^{*}\right)-p^{*}\left(u^{*} ; a^{*}\right) \\
& =p(v ; a)-p(u ; a)+\sum_{\mu=1}^{n} k_{\mu}(u, v)\left[\omega_{\mu}(a)-\omega_{\mu}^{*}\left(a^{*}\right)\right] \\
& \quad+e^{i \phi} \rho^{2} Q^{\prime}\left(z_{0} ; u, v\right) p^{\prime}\left(z_{0} ; a\right)+e^{-i \phi} \rho^{2}\left(P^{\prime}\left(z_{0} ; u, v\right)\right)^{+}\left(p^{\prime}\left(z_{0} ; a\right)\right)^{+}+c\left(\rho^{2}\right) .
\end{aligned}
$$

By virtue of (169) we have, therefore, for the domain function

$$
D(v, u ; a)=p(v ; a)-p(u ; a),
$$

the variational equation

$$
\begin{aligned}
& D^{*}\left(v^{*}, u^{*} ; a^{*}\right) \\
& =D(v, u ; a)+e^{i \phi} \rho^{2}\left[Q^{\prime}\left(z_{0} ; u, v\right)-\frac{1}{2} \sum_{\mu=1}^{n} k_{\mu}(u, v) w_{\mu}^{\prime}\left(z_{0}\right)\right] p^{\prime}\left(z_{0} ; a\right) \\
& \quad+e^{-i \phi} \rho^{2}\left[\left(P^{\prime}\left(z_{0} ; u, v\right)\right)^{+}-\frac{1}{2} \sum_{\mu=1}^{n} k_{\mu}(u, v)\left(w_{\mu}^{\prime}\left(z_{0}\right)\right)^{+}\right]\left(p^{\prime}\left(z_{0} ; a\right)\right)^{+} \\
& \quad+o\left(\rho^{2}\right) .
\end{aligned}
$$

Using again the notation $\left(172^{\prime \prime}\right)$ and (103) we bring this result into the final form 


$$
\begin{aligned}
D^{*}\left(v^{*}, u^{*} ; a^{*}\right)= & D(v, u ; a)+e^{i \phi} \rho^{2} p^{\prime}\left(z_{0} ; a\right) \frac{\partial}{\partial z_{0}} D\left(v, u ; z_{0}\right) \\
& +e^{-i \phi} \rho^{2}\left(p^{\prime}\left(z_{0} ; a\right)\right)+\frac{\partial}{\partial z_{0}^{+}} D\left(v, u ; z_{0}\right)+o\left(\rho^{2}\right) .
\end{aligned}
$$

If we are interested only in the variation of the real part of $D(v, u ; a)$ we may derive from $\left(172^{\prime \prime \prime}\right)$ the simpler formula

$$
\begin{aligned}
& \operatorname{Re}\left\{D\left(v^{*}, u^{*} ; a^{*}\right)-D(v, u ; a)\right\} \\
& =\operatorname{Re}\left\{e ^ { i \phi } \rho ^ { 2 } p ^ { \prime } ( z _ { 0 } ; a ) \left[Q^{\prime}\left(z_{0} ; u, v\right)+P^{\prime}\left(z_{0} ; u, v\right)\right.\right. \\
& \left.\left.\quad-\frac{1}{2} \sum_{\mu=1}^{n} w_{\mu}^{\prime}\left(z_{0}\right)\left(k_{\mu}(u, v)+\left(k_{\mu}(u, v)\right)^{+}\right)\right]\right\}+o\left(\rho^{2}\right) .
\end{aligned}
$$

Now one finds easily from (75) and (78) that

$$
\frac{1}{2} \sum_{\mu=1}^{n} w_{\mu}^{\prime}\left(z_{0}\right)\left[k_{\mu}(u, v)+\left(k_{\mu}(u, v)\right)^{+}\right]=\sum_{\nu=1}^{n}\left[\omega_{\nu}(u)-\omega_{\nu}(v)\right] l_{\nu}\left(z_{0}\right),
$$

whence by use of (61), (63) and $\left(103^{\prime}\right)$ after easy transformations

$$
\begin{aligned}
g^{*}\left(v^{*} ; a^{*}\right)-g^{*}\left(u^{*} ; a^{*}\right) & =g(v ; a)-g(u ; a) \\
+ & \operatorname{Re}\left\{e^{i \phi} \rho^{2} p^{\prime}\left(z_{0} ; a\right)\left[p^{\prime}\left(z_{0} ; v\right)-p^{\prime}\left(z_{0} ; u\right)\right]\right\}+o\left(\rho^{2}\right) .
\end{aligned}
$$

Sending finally $u$ to the boundary $C$ of $D$ one obtains

$$
g^{*}\left(v^{*} ; a^{*}\right)=g(v ; a)+\operatorname{Re}\left\{e^{i \phi} \rho^{2} p^{\prime}\left(z_{0} ; a\right) p^{\prime}\left(z_{0} ; v\right)\right\}+o\left(\rho^{2}\right) .
$$

This is a known variation formula for Green's function [10, 12].

Subtracting the conjugate of equation (166) from equation (164) and using (108), we obtain a variation formula for Neumann's function:

$$
\begin{aligned}
\pi^{*}\left(b^{*} ; u^{*}\right)-\pi^{*}\left(a^{*} ; u^{*}\right)-\pi^{*}\left(b^{*} ; v^{*}\right)+\pi^{*}\left(a^{*} ; v^{*}\right) \\
=\pi(b ; u)-\pi(a ; u)-\pi(b ; v)+\pi(a ; v) \\
\quad-e^{i \phi} \rho^{2} Q^{\prime}\left(z_{0} ; a, b\right)\left[Q^{\prime}\left(z_{0} ; u, v\right)-P^{\prime}\left(z_{0} ; u, v\right)\right] \\
\quad+e^{-i \phi} \rho^{2}\left(P^{\prime}\left(z_{0} ; a, b\right)\right)^{+}\left[\left(Q^{\prime}\left(z_{0} ; u, v\right)\right)^{+}-\left(P^{\prime}\left(z_{0} ; u, v\right)\right)^{+}\right]+o\left(\rho^{2}\right),
\end{aligned}
$$

which leads by application of $\left(108^{\prime}\right),(61)$ and (63) to

$$
\begin{aligned}
\pi^{*}\left(b^{*} ; u^{*}\right)-\pi^{*}\left(a^{*} ; u^{*}\right)-\pi^{*}\left(b^{*} ; v^{*}\right)+\pi^{*}\left(a^{*} ; v^{*}\right) \\
=\pi(b ; u)-\pi(a ; u)-\pi(b ; v)+\pi(a ; v) \\
\quad-e^{i \phi} \rho^{2} Q^{\prime}\left(z_{0} ; a, b\right)\left[\pi^{\prime}\left(z_{0} ; v\right)-\pi^{\prime}\left(z_{0} ; u\right)\right] \\
\quad+e^{-i \phi} \rho^{2}\left(P^{\prime}\left(z_{0} ; a, b\right)\right)^{+}\left[\left(\pi^{\prime}\left(z_{0} ; v\right)\right)^{+}-\left(\pi^{\prime}\left(z_{0} ; u\right)\right)^{+}\right]+o\left(\rho^{2}\right) .
\end{aligned}
$$

Let us introduce the domain function $\log \Psi(z ; u, v)$, defined in (99). Con- 
sidering only the real parts in $\left(176^{\prime}\right)$, we obtain

$$
\begin{aligned}
& \log \left|\Psi^{*}\left(b^{*} ; u^{*}, v^{*}\right)\right|-\log \left|\Psi^{*}\left(a^{*} ; u^{*}, v^{*}\right)\right| \\
& =\log |\Psi(b ; u, v)|-\log |\Psi(a ; u, v)| \\
& \quad+\operatorname{Re}\left\{e^{i \phi} \rho^{2} \frac{\partial}{\partial z_{0}} \log \Psi\left(z_{0} ; u, v\right) \frac{\partial}{\partial z_{0}} \log \Psi\left(z_{0} ; a, b\right)\right\}+o\left(\rho^{2}\right) .
\end{aligned}
$$

Numerous similar identities may be obtained by the same method. We applied in $\$ 3$ the method of contour integration by pairing different domain functions of $D$. If one of the two domain functions is replaced by the corresponding domain function with respect to $D^{*}$, we get a variation formula. There is a similar procedure for Riemann surfaces which makes it possible to obtain variation formulas for the normal integrals and their periods by contour integration [12].

The variation formula $\left(175^{\prime}\right)$ might have been obtained also by the following application of our formal identities. Suppose that $g^{*}(z ; a)$ is Green's function for the domain $D^{*}$, obtained by the variation (157) from the original domain $D$. If $z$ and $a$ are different from $z_{0}$ we have by Taylor's formula

$$
g^{*}\left(z^{*} ; a^{*}\right)=g^{*}(z ; a)+\operatorname{Re}\left\{e^{i \phi} \rho^{2}\left(\frac{p^{\prime}(z ; a)}{z-z_{0}}+\frac{p^{\prime}(a ; z)}{a-z_{0}}\right)\right\}+o\left(\rho^{2}\right) .
$$

The first two terms on the right-hand side represent a harmonic function of $z$ in $D$, except for the point $z_{0}$, where it has a simple pole, and the point $a$, where the first term has a logarithmic pole but the second term remains finite. If $z$ lies on $C$ we have further because of (178) small values of magnitude $o\left(\rho^{2}\right)$ for this expression. We shall now add to this function another harmonic function of $z$ which has the boundary values 0 on $C$ and cancels just the singularity of the second term at $z_{0}$. The sum function is then harmonic everywhere in $D$, except for the logarithmic pole at $a$, and has on $C$ the order of magnitude $o\left(\rho^{2}\right)$. It is, therefore, up to a corrective term of order $o\left(\rho^{2}\right)$ identical with Green's function. The new harmonic function is

$$
H\left(z, a ; z_{0}\right)=-\operatorname{Re}\left\{e^{i \phi} \rho^{2} p^{\prime}\left(z_{0} ; z\right) p^{\prime}\left(z_{0} ; a\right)\right\} .
$$

In fact, because of $\left(103^{\prime}\right)$ and $(57)$ one has

$$
p^{\prime}(w ; z) \equiv 0
$$
for $w \in D, z \in C$.

Hence, this harmonic function vanishes on $C$ and one finds by inspection that it cancels the singularity of the second right-hand term in (178). We obtain finally

$$
\begin{aligned}
\left(178^{\prime \prime \prime}\right) & g^{*}(z ; a)+\operatorname{Re}\left\{e^{i \phi} \rho^{2}\left[\frac{p^{\prime}(z ; a)}{z-z_{0}}+\frac{p^{\prime}(a ; z)}{a-z_{0}}-p^{\prime}\left(z_{0} ; z\right) p^{\prime}\left(z_{0} ; a\right)\right]\right\} \\
& =g(z ; a)+o\left(\rho^{2}\right) .
\end{aligned}
$$


This consideration shows the close connection between a variational problem and the problem of constructing a harmonic function with prescribed singularities which vanishes at the boundary of the domain. This is just the same problem which arises in the theory of the Schottky functions and it becomes obvious why the same method applies to these different fields.

7. Restricted variations. In the theory of domain functions one has sometimes to investigate an extremum problem in a class of domains which is defined by certain normalization conditions. If one wants to attack such a problem by variational methods, the question arises how to carry out the variation within the restricted class of domains considered. This is analogous to the case of isoperimetric problems in the classical calculus of variations, but may be much more difficult. Our results enable us now to overcome these difficulties in the case of two important types of restriction.

A typical case of the first type is the following. We wish to vary the domain $D$ bounded by the continua $C_{1}, \cdots, C_{n}$ without changing the conformal type by means of a variation of the form

$$
z^{*}=z+\sum_{t=1}^{N} \frac{a_{t} \rho^{2}}{z-z_{t}}, \quad \rho>0,\left|a_{t}\right| \leqq 1,
$$

to be understood in the same sense as the variation (157).

We assume that $N \geqq 3 n-6$, which is the number of conformal moduli of the domain, and we try to find the conditions upon the parameters $a_{t}$, after the $z_{t}$ have been chosen, so that the conformal type of the domain is unchanged. For this purpose we merely pick a set of moduli, determine their variations by the methods of $\$ 6$, and set these equal to zero.

A convenient set of moduli is obtained by use of a fixed linear combination $w(z)$ of the harmonic measures with real coefficients. We take $n-1$ of the periods of $w(z)$, the values of $\omega(z)=\operatorname{Re}\{w(z)\}$ at the $n-2$ critical points $d_{1}, \cdots, d_{n-2}$ of $w(z)$ and the $n-3$ differences of $\operatorname{Im}\{w(z)\}$ at the pairs of critical points $\left(d_{2}, d_{1}\right), \cdots,\left(d_{n-2}, d_{1}\right)$. These invariants are moduli, since if they are the same for two domains $D$ and $D^{*}$, then the implicit equation

$$
w^{*}\left(z^{*}\right)=w(z)
$$

can be solved to yield the map $z^{*}(z)$ of $D$ upon $D^{*}$. Here the asterisks refer to quantities associated with $D^{*}$.

To obtain the variations of our moduli so chosen, we note that they are linear functions in the coefficients $a_{t}$ and recall (169). By application of (103')

$$
\begin{aligned}
\omega^{*}\left(z^{*}\right)=\omega(z)+\operatorname{Re}\left\{\sum _ { t = 1 } ^ { N } a _ { t } \rho ^ { 2 } w ^ { \prime } ( z _ { l } ) \left[N\left(z ; z_{t}\right)-\left(M\left(z ; z_{t}\right)\right)^{+}\right.\right. & \\
& \left.\left.+\sum_{\nu=1}^{n} l_{\nu}\left(z_{t}\right) \omega_{\nu}(z)\right]\right\}+o\left(\rho^{2}\right),
\end{aligned}
$$


whence we obtain for the analytic functions $w^{*}\left(z^{*}\right)$ and $w(z)$

$$
\begin{aligned}
w^{*}\left(z^{*}\right)=w(z) & +\sum_{t=1}^{N} a_{t} \rho^{2} w^{\prime}\left(z_{t}\right) N\left(z ; z_{t}\right)-\sum_{t=1}^{N} a_{t}^{+} \rho^{2}\left(w^{\prime}\left(z_{t}\right)\right)+M\left(z ; z_{t}\right) \\
& +\sum_{t=1}^{N} \sum_{\nu=1}^{n} \operatorname{Re}\left\{a_{t} \rho^{2} w^{\prime}\left(z_{t}\right) l_{\nu}\left(z_{t}\right)\right\} w_{\nu}(z)+i K+o\left(\rho^{2}\right),
\end{aligned}
$$

where $K$ is some real constant. Since $N\left(z ; z_{t}\right)$ and $M\left(z ; z_{t}\right)$ have no periods, we see that the conditions for the invariance of the $n-1$ periods of $w(z)$ which we have chosen are

$$
\operatorname{Re}\left\{\sum_{t=1}^{N} a_{t} w^{\prime}\left(z_{t}\right) w_{\mu}^{\prime}\left(z_{t}\right)\right\}=o(1), \quad \mu=1,2, \cdots, n-1,
$$

upon application of (78). This is, of course, also a direct consequence of (170). Letting $d_{\mu}^{*}$ be the critical points of $\omega^{*}\left(z^{*}\right)$, we verify that

$$
\omega^{*}\left(d_{\mu}^{*}\right)=\omega^{*}\left(\tilde{d}_{\mu}\right)+o\left(\rho^{2}\right), \quad \quad \tilde{d}_{\mu}=z^{*}\left(d_{\mu}\right) .
$$

Hence we can conclude from (169) that the $\omega\left(d_{\mu}\right)$ are invariant when

$$
\operatorname{Re}\left\{\sum_{t=1}^{N} a_{t} w^{\prime}\left(z_{t}\right) p^{\prime}\left(z_{t} ; d_{\mu}\right)\right\}=o(1), \quad \mu=1,2, \cdots, n-2 .
$$

Finally we note that when (181) is satisfied, the third term on the right in $\left(169^{\prime \prime}\right)$ must be constant because of (78) and the character of the $P_{\mu \nu}$-matrix. Hence, under these circumstances we find for the remaining $n-3$ moduli the conditions of invariance

$$
\begin{aligned}
\operatorname{Im}\left\{\sum_{t=1}^{N} a_{t} w^{\prime}\left(z_{t}\right)\left[\pi^{\prime}\left(z_{t} ; d_{\mu}\right)-\pi^{\prime}\left(z_{t} ; d_{1}\right)\right]\right\}= & o(1), \\
& \mu=2,3, \cdots, n-2,
\end{aligned}
$$

by use of $\left(108^{\prime}\right)$ in transforming $\left(169^{\prime \prime}\right)$. The constant $K$ drops out, since we take a difference $\operatorname{Im}\left\{w\left(d_{\mu}\right)-w\left(d_{1}\right)\right\}$ in each case. The $3 n-6$ conditions (181), (183) and (184) are, then, necessary and sufficient that the variation (179) leave the conformal type of $D$ unchanged, for suitably chosen terms $o(1)$.

If the determinant of the coefficients of $a_{1}, \cdots, a_{3 n-6}$ does not vanish identically for $N=3 n-6$ in the equations (181), (183) and (184), then by the topological analogue of the implicit function theorem we may find numbers $a_{t}$ so that these equations are actually fulfilled. The determinant will not vanish identically provided that the functions $w_{1}^{\prime}(z), \cdots, w_{n-1}^{\prime}(z)$, $p^{\prime}\left(z, d_{1}\right), \cdots, p^{\prime}\left(z, d_{n-2}\right),\left\{\pi^{\prime}\left(z, d_{2}\right)-\pi^{\prime}\left(z, d_{1}\right)\right\}, \cdots,\left\{\pi^{\prime}\left(z, d_{n-2}\right)-\pi^{\prime}\left(z, d_{1}\right)\right\}$ are linearly independent. Since $\pi^{\prime}\left(z, a_{\mu}\right)$ and $p^{\prime}\left(z, d_{\mu}\right)$ are the only functions in this set with poles at $d_{\mu}$, a linear dependence relation must have the form 


$$
\begin{aligned}
L^{\prime}(z)= & \sum_{\mu=1}^{n-1} \alpha_{\mu} w_{\mu}^{\prime}(z) \\
& +\sum_{\mu=2}^{n-2} \beta_{\mu}\left[\pi^{\prime}\left(z ; d_{\mu}\right)-\pi^{\prime}\left(z ; d_{1}\right)-p^{\prime}\left(z ; d_{\mu}\right)+p^{\prime}\left(z ; d_{1}\right)\right] \equiv 0 .
\end{aligned}
$$

Thus for any function $f(z)$ regular in $D$ and smooth on $C$

$$
\begin{aligned}
0= & \oint_{C} f(z)\left(L^{\prime}(z)\right)^{+} d z^{+}=-\sum_{\mu=1}^{n-1} \alpha_{\mu}^{+} \oint_{C} f(z) d w_{\mu}(z) \\
& +\sum_{\mu=2}^{n-2} \beta_{\mu}^{+} \oint_{C} f(z)\left[\pi^{\prime}\left(z ; d_{\mu}\right)-\pi^{\prime}\left(z ; d_{1}\right)+p^{\prime}\left(z ; d_{\mu}\right)-p^{\prime}\left(z ; d_{1}\right)\right] d z \\
= & -4 \pi i \sum_{\mu=2}^{n-2} \beta_{\mu}^{+}\left[f\left(d_{\mu}\right)-f\left(d_{1}\right)\right]
\end{aligned}
$$

by our usual device. Since $\left[f\left(d_{\mu}\right)-f\left(d_{1}\right)\right]$ may be chosen arbitrarily, we have $\beta_{\mu}=0, \mu=2, \cdots, n-2$, and it follows from the linear independence of the $w_{\mu}^{\prime}(z)$ that $\alpha_{\mu}=0, \mu=1, \cdots, n-1$. Thus we have the required independence and our result is completely proved. The points in the proof where we use smoothness of $C$ do not require this assumption in any essential way, so that we can take $C$ to consist of any $n$ proper continua.

If we wish to consider extremum problems for conformal mappings leaving, say, the point at infinity in $D$ fixed, we may derive from the Green's function $p(z, \infty)$ and the corresponding system of moduli in this case the $3 n-4$ conditions of invariance of restricted conformal type

$$
\begin{array}{ll}
\operatorname{Re}\left\{\sum_{t=1}^{N} a_{t} p^{\prime}\left(z_{t} ; \infty\right) w_{\mu}^{\prime}\left(z_{t}\right)\right\}=o(1), & \mu=1,2, \cdots, n-1, \\
\operatorname{Re}\left\{\sum_{t=1}^{N} a_{t} p^{\prime}\left(z_{t} ; \infty\right) p^{\prime}\left(z_{t} ; d_{\mu}\right)\right\}=o(1), & \mu=1,2, \cdots, n-1, \\
\operatorname{Im}\left\{\sum_{t=1}^{N} a_{t} p^{\prime}\left(z_{t} ; \infty\right)\left[\pi^{\prime}\left(z_{t} ; d_{\mu}\right)-\pi^{\prime}\left(z_{t} ; d_{1}\right)\right]\right\}=o(1), \\
\mu=2,3, \cdots, n-1,
\end{array}
$$

where the $d_{\mu}$ are the $n-1$ finite critical points of $p(z, \infty)$. Similar conditions may be derived for the original Hadamard variational formulas which give the variation of the domain functions under an arbitrary variation of the boundary [12].

We see from our work on the functions $w_{i}^{\prime} w_{k}^{\prime}$ at the end of $\S 5$ that in general it will be sufficient to set the variations of the $P_{i k}$ equal to zero to obtain a type-invariant variation. For by the methods of that section we can easily show that every domain function $f(z)$ for which $f(z) z^{\prime 2}$ is real on $C$ and 
which is regular in $D$ may be composed linearly from $3 n-6$ linearly independent products $w_{i}^{\prime}(z) w_{k}^{\prime}(z)$. Now the analytic functions appearing in (181), (183), (184) are just of this type and, on the other hand, the variations of the $P_{i k}$ are expressed in terms of the $w_{i}^{\prime} w_{k}^{\prime}$.

We solve a particular well known problem by this variational method in order to show how such proofs go. Consider the domain $\widetilde{D}$ bounded by $C_{1}$ and $C_{2}$, where $C_{1}$ is assumed to be the outer boundary of $D$. In general the conformal type of $\widetilde{D}$ will change when $D$ is mapped conformally, and we minimize the modulus of $\widetilde{D}$ for all conformal images of $D$. The mappings of $D$ may be restricted in any number of ways to give a normal family without changing the problem, for example by fixing the derivative at a point. We take the modulus to be the period $\widetilde{P}_{11}$ of the harmonic measure of $C_{1}$ in any domain $\widetilde{D}$ obtained by mapping $D$.

We make a variation (179) of the extremal domain $D$ satisfying (181), (183) and (184) and obtain from the minimum property of $\widetilde{P}_{11}$ the inequality

$$
\tilde{P}_{11}^{*}-\tilde{P}_{11}=\operatorname{Re}\left\{\sum_{t=1}^{N} a_{t} \rho^{2} \tilde{w}_{1}^{\prime}\left(z_{t}\right)^{2}\right\}+o\left(\rho^{2}\right) \geqq 0,
$$

where $\tilde{w}_{1}(z)$ is the harmonic measure function of $C_{1}$ in $\tilde{D}$.

The reader can verify by the familiar procedure leading to Lagrange multipliers in the calculus of variations that this implies the existence of real numbers $\lambda_{\mu}$ such that

$$
\begin{aligned}
\tilde{w}_{1}^{\prime}(z)^{2}= & \sum_{\mu=1}^{n-1} \lambda_{\mu} w^{\prime}(z) w_{\mu}^{\prime}(z)+\sum_{\mu=1}^{n-2} \lambda_{\mu+n-1} w^{\prime}(z) p^{\prime}\left(z ; d_{\mu}\right) \\
& +i \sum_{\mu=2}^{n-2} \lambda_{\mu+2 n-4} w^{\prime}(z)\left[\pi^{\prime}\left(z ; d_{\mu}\right)-\pi^{\prime}\left(z ; d_{1}\right)\right] .
\end{aligned}
$$

It follows from this relation that the boundary components $C_{\nu}, \nu>2$, are analytic curves $z_{\nu}(s)$ satisfying the differential equations

$$
\begin{aligned}
\tilde{w}_{1}^{\prime}(z)^{2} \cdot z_{\nu}^{\prime}(s)^{2}= & \sum_{\mu=1}^{n-1} \lambda_{\mu} \frac{\partial w}{\partial s} \frac{\partial w_{\mu}}{\partial s}+\sum_{\mu=1}^{n-2} \lambda_{\mu+n-1} \frac{\partial w}{\partial s} \frac{\partial p\left(z ; d_{\mu}\right)}{\partial s} \\
& +i \sum_{\mu=2}^{n-2} \lambda_{\mu+2 n-4} \frac{\partial w}{\partial s}\left[\frac{\partial \pi\left(z ; d_{\mu}\right)}{\partial s}-\frac{\partial \pi\left(z ; d_{1}\right)}{\partial s}\right]=\text { real. }
\end{aligned}
$$

For each separate arc we can bring this into the form

$$
\left[\partial \tilde{w}_{1}\left(z_{\nu}(t)\right) / \partial t\right]^{2}= \pm 1
$$

by suitable choice of the real parameter $t$. Now there is a map of each small arc $C_{\rho}$ of mapping radius $\rho$ of $C_{\nu}, \nu>2$, on the exterior of a circle of radius $\rho$, of the form

$$
z^{*}=z+a \rho^{2} /\left(z-z_{0}\right)+b \rho^{3} /\left(z-z_{0}\right)^{2}+\cdots, \quad z_{0} \in C_{\rho}
$$


with uniformly bounded coefficients $a, b, \ldots$ In each such map we obtain from (170) and the minimum property of $D$

$$
\operatorname{Re}\left\{a \rho^{2} \tilde{w}_{1}^{\prime}\left(z_{0}\right)^{2}\right\}+o\left(\rho^{2}\right) \geqq 0 .
$$

If the curve $C_{\rho}$ is rotated until it has a horizontal tangent at $z_{0}$, we can choose $a=-1+o(1)$ and we find

$$
\left(190^{\prime \prime}\right) \quad \operatorname{Re}\left\{\tilde{w}_{1}^{\prime}\left(z_{0}\right)^{2}\right\} \leqq 0 .
$$

Hence the lower sign must hold in our differential equations and we have

$$
\tilde{w}_{1}\left(z_{\nu}(t)\right)= \pm i t+\text { const., }
$$

so that the $C_{\nu}$ lie along the level curves of $\tilde{\omega}_{1}(z)$. We remark that $\widetilde{P}_{11}$ is unchanged by conformal mappings of $\tilde{D}$, which are also mappings of $D$, and that in consequence there is no loss of generality in assuming $C_{1}$ and $C_{2}$ to be concentric circles. In this case the $C_{\nu}, \nu>2$, are concentric circular slits and the extremal map is a function $f_{12}(z)$ of the type introduced in $\$ 2$.

Our progress, then, has been to find that for the extremal domain the $C_{\nu}, \nu>2$, are analytic curves without use of the boundary variation (194) introduced earlier [9]. We then use the boundary variation to complete the result, but in a very simple form. Thus we have reduced the work involved in the application of variational methods to a considerably more elementary and less involved set of ideas.

We come now to the second type of restricted variational problems which we desire to handle. Given are $n$ closed smooth curves $C_{\boldsymbol{v}}$ which are the boundary of a domain $D$ in the complex plane which contains the point at infinity. We seek a continuum containing all the curves $C_{\nu}$ of minimum capacity. We require of variations considered that they transform each continuum $C_{\nu}$ into itself. This is, in fact, possible to obtain by means of variations

$$
z^{*}=z+\left(e^{i \phi} \rho^{2} Q^{\prime}(z ; u, v)+e^{-i \phi} \rho^{2} P^{\prime}(z ; u, v)\right)\left(w_{i}^{\prime}(z) w_{k}^{\prime}(z)\right)^{-1}+o\left(\rho^{2}\right) .
$$

According to (16) we have, namely, in $C$

$$
z^{\prime} Q^{\prime}(z ; u, v) e^{i \phi}+z^{\prime} P^{\prime}(z ; u, v) e^{-i \phi}=\text { real, } \quad z^{\prime}=d z / d s .
$$

Since further $w_{i}^{\prime}(z) z^{\prime}$ and $w_{k}^{\prime}(z) z^{\prime}$ are imaginary on $C$, one sees that $z^{*}-z$ has the direction of the tangent vector at $z$ to $C$, up to a term $o\left(\rho^{2}\right)$. By a correction term $o\left(\rho^{2}\right)$ it is possible to attain a complete preservation of the contours $C_{\nu}$. This can be seen by making a variation

$$
z^{* *}=z^{*}+\sum_{t=1}^{N} \frac{a_{t}}{z^{*}-z_{t}} \cdot o\left(\rho^{2}\right)
$$

to bring the conformal type of our domain back to that of the original region, and then it is clear that there is a conformal map 


$$
z^{* * *}=z^{* *}+o\left(\rho^{2}\right)
$$

of the domain of the $z^{* *}$-plane back upon the original domain. For fixed $w(z)$ we have a freedom of choice of $u$ and $v$ in $D$ and of arbitrary real $\phi$ and $\rho$. Thus, this type of variation permits the usual applications in extremum problems. We proceed to the complete solution of the above-mentioned problem.

Let $\tilde{D}$ be the domain bounded by the minimizing continuum, and let $\tilde{p}(z, \infty)$ be the corresponding analytic function for Green's function with pole at infinity. The function $\tilde{g}(z, \infty)$ has at $\infty$ an expansion

$$
\tilde{g}(z ; \infty)=\log |z|+\gamma+o(1),
$$

and the capacity of our continuum is by definition $e^{-\gamma}$. Hence we obtain upon making our variation the inequality

$$
\begin{aligned}
0 \geqq & \gamma^{*}-\gamma=\operatorname{Re}\left\{e^{i \phi} \rho^{2}\left[\frac{\tilde{p}^{\prime}(u ; \infty)^{2}}{w_{i}^{\prime}(u) w_{k}^{\prime}(u)}-\frac{\tilde{p}^{\prime}(v ; \infty)^{2}}{w_{i}^{\prime}(v) w_{k}^{\prime}(v)}\right]\right. \\
& +\sum_{\mu=1}^{n-2} \frac{e^{i \phi} \rho^{2} Q^{\prime}\left(d_{i \mu} ; u, v\right)+e^{-i \phi} \rho^{2} P^{\prime}\left(d_{i \mu} ; u, v\right)}{w_{i}^{\prime \prime}\left(d_{i \mu}\right) w_{k}^{\prime}\left(d_{i \mu}\right)} \tilde{p}^{\prime}\left(d_{i \mu} ; \infty\right)^{2} \\
& \left.+\sum_{\mu=1}^{n-2} \frac{e^{i \phi} \rho^{2} Q^{\prime}\left(d_{k \mu} ; u, v\right)+e^{-i \phi} \rho^{2} P^{\prime}\left(d_{k \mu} ; u, v\right)}{w_{i}^{\prime}\left(d_{k \mu}\right) w_{k}^{\prime \prime}\left(d_{k \mu}\right)} \tilde{p}^{\prime}\left(d_{k \mu} ; \infty\right)^{2}\right\}+o\left(\rho^{2}\right),
\end{aligned}
$$

where the $d_{i \mu}$ are the critical points of $w_{i}(z)$ and the $d_{k \mu}$ are the critical points of $w_{k}(z)$. By the arbitrariness of $\phi$ and $\rho$ and from the relations (61) and (63),

$$
\begin{aligned}
& \frac{\tilde{p}^{\prime}(u ; \infty)^{2}}{w_{i}^{\prime}(u) w_{k}^{\prime}(u)}-\sum_{\mu=1}^{n-2}\left[\frac{\tilde{p}^{\prime}\left(d_{i \mu} ; \infty\right)^{2}}{w_{i}^{\prime \prime}\left(d_{i \mu}\right) w_{k}^{\prime}\left(d_{i \mu}\right)} N\left(u ; d_{i \mu}\right)\right. \\
& \left.\quad-\left(\frac{\tilde{p}^{\prime}\left(d_{i \mu} ; \infty\right)^{2}}{w_{i}^{\prime \prime}\left(d_{i \mu}\right) w_{k}^{\prime}\left(d_{i \mu}\right)}\right)^{+} M\left(u ; d_{i \mu}\right)\right] \\
& \quad-\sum_{\mu=1}^{n-2}\left[\frac{\tilde{p}^{\prime}\left(d_{k \mu} ; \infty\right)^{2}}{w_{i}^{\prime}\left(d_{k \mu}\right) w_{k}^{\prime \prime}\left(d_{k \mu}\right)} N\left(u ; d_{k \mu}\right)-\left(\frac{\tilde{p}^{\prime}\left(d_{k \mu} ; \infty\right)^{2}}{w_{i}^{\prime}\left(d_{k \mu}\right) w_{k}^{\prime \prime}\left(d_{k \mu}\right)}\right)^{+} M\left(u ; d_{k \mu}\right)\right] \\
& =\frac{\tilde{p}^{\prime}(v ; \infty)^{2}}{w_{i}^{\prime}(v) w_{k}^{\prime}(v)}-\sum_{\mu=1}^{n-2}\left[\frac{\tilde{p}^{\prime}\left(d_{i \mu} ; \infty\right)^{2}}{w_{i}^{\prime \prime}\left(d_{i \mu}\right) w_{k}^{\prime}\left(d_{i \mu}\right)} N\left(v ; d_{i \mu}\right)\right. \\
& \left.\quad-\left(\frac{\tilde{p}^{\prime}\left(d_{i \mu} ; \infty\right)^{2}}{w_{i}^{\prime \prime}\left(d_{i \mu}\right) w_{k}^{\prime}\left(d_{i \mu}\right)}\right)^{+} M\left(v ; d_{i \mu}\right)\right] \\
& \quad-\sum_{\mu=1}^{n-2}\left[\frac{\tilde{p}^{\prime}\left(d_{k \mu} ; \infty\right)^{2}}{w_{i}^{\prime}\left(d_{k \mu}\right) w_{k}^{\prime \prime}\left(d_{k \mu}\right)} N\left(v ; d_{k \mu}\right)-\left(\frac{\tilde{p}^{\prime}\left(d_{k \mu} ; \infty\right)^{2}}{w_{i}^{\prime}\left(d_{k \mu}\right) w_{k}^{\prime \prime}\left(d_{k \mu}\right)}\right)^{+} M\left(v ; d_{k \mu}\right)\right] \\
& =\text { const., }
\end{aligned}
$$$$
\left(200^{\prime}\right)
$$

since each side of the equation is a function of a different variable. We conclude that $\tilde{p}^{\prime}(u ; \infty)^{2}$ is analytic throughout $D$; in fact we have shown that 


$$
\tilde{p}^{\prime}(u ; \infty)^{2} / w_{i}^{\prime}(u) w_{k}^{\prime}(u)=S(u),
$$

where $S(u)$ is a Schottky function in $D$.

From the fact that $\widetilde{p}^{\prime}(u ; \infty)^{2}$ is analytic in $D$, we can conclude that each slit $\tilde{C}_{\alpha}$ of the boundary of $\tilde{D}$ in $D$ is an analytic curve, and that in the mapping of $D$ upon the unit circle taking the point at infinity into the origin each side of any segment of the slit $\tilde{C}_{\alpha}$ is taken into an arc of the same length as that of the image of the other side of the slit. This is because the distortion of the mapping on the boundary $\tilde{C}_{\alpha}$ is in both cases $\left|\tilde{p}^{\prime}(z ; \infty)\right|$. The function $\tilde{p}^{\prime}(z ; \infty)$ can be extended to be analytic on a two-sheeted Riemann surface covering $D$; it assumes values symmetric in the origin at points lying over one another. From these facts one can carry out a further discussion of the nature of the $\tilde{C}_{\alpha}$.

We remark that this problem could be solved equally well with the restricted variation leaving $D$ conformally invariant for maps preserving the point at infinity with fixed distortion. But this method would have led us to a set of Lagrange multipliers which are determined explicitly by the tangential variation in terms of the various domain functions. Thus the importance of the relations we have derived becomes apparent.

As a final application of our identities we derive the conditions for unchanged conformal type under variations (179) from a second point of view in the case of a smoothly bounded domain $D$. We start from the assumption that the parameters $a_{t}, z_{t}$ have been chosen in the right way and that $D$ is mapped upon a domain $D^{*}$ which is conformally equivalent to it. There exists, therefore, a univalent function in $D$ which maps $D$ upon $D^{*}$. Let

$$
\zeta=z+\rho^{2} k_{\rho}(z)
$$

be the mapping function, where the factor $\rho^{2}$ measures the order of the second term. If we invert (179) into

$$
z_{1}=v^{-1}\left(z^{*}\right)=z^{*}-\sum_{t=1}^{N} \frac{a_{t} \rho^{2}}{z^{*}-z_{t}}+o\left(\rho^{2}\right)
$$

this gives a map of $D^{*}$ upon $D$ except for a certain neighborhood of the $z_{t} \in D^{*}$. In particular, the boundary $C^{*}$ of $D^{*}$ will be transformed into the boundary of $D$ by means of $\left(179^{\prime}\right)$. Therefore, the composite map

$$
v^{-1}(\zeta)=z+\rho^{2} k_{\rho}(z)-\sum_{t=1}^{N} \frac{a_{t} \rho^{2}}{z-z_{t}}+o\left(\rho^{2}\right)
$$

transforms each $C_{v}$ into itself. Thus, having chosen $a_{t}$ and $z_{t}$ in the right way, we have in the limit $\rho \rightarrow 0$ the following condition on $C$ :

$$
\left(\lim _{\rho \rightarrow 0} k_{\rho}(z)-\sum_{t=1}^{N} \frac{a_{t}}{z-z_{t}}\right) z^{\prime-1}=\text { real, } \quad z^{\prime}=\frac{d z}{d s} .
$$

On the other hand, if we can determine a function $k(z)$ which is regular in 
$D+C$ and such that

$$
k(z)-\sum_{t=1}^{N} \frac{a_{t}}{z-z_{t}}=\lambda(z) z^{\prime}, \quad \lambda(z)=\text { real, } z \in C,
$$

we can chose a correction term $o\left(\rho^{2}\right)$ so that

$$
z^{*}=z+\sum_{t=1}^{N} \frac{a_{t} \rho^{2}}{z-z_{t}}+o\left(\rho^{2}\right)
$$

maps $D$ upon a conformally equivalent domain $D^{*}$.

Thus our problem is reduced to the determination of a function $k(z)$ of the required type. Choose for this purpose a function $w^{\prime}(z)$ of type (143); evidently

$$
f(z)=\frac{1}{i} w^{\prime}(z)\left[k(z)-\sum_{t=1}^{N} \frac{a_{t}}{z-z_{t}}\right]
$$

is a Schottky function. It has simple poles at the $N$ points $z_{t}$ and the condition (125) connecting poles and residues of a function of class $\Re$ yields

$$
\operatorname{Re}\left\{\sum_{t=1}^{N} a_{t} w^{\prime}\left(z_{t}\right) w_{\mu}^{\prime}\left(z_{t}\right)\right\}=0, \quad \quad \mu=1,2, \cdots, n-1 .
$$

Now we may apply the representation (122) for $f(z)$ and find

$$
\begin{aligned}
\frac{1}{i} w^{\prime}(z)[k(z) & \left.-\sum_{t=1}^{N} \frac{a_{t}}{z-z_{t}}\right] \\
& =A-\frac{1}{i} \sum_{t=1}^{N}\left(a_{t} w^{\prime}\left(z_{t}\right) N\left(z ; z_{t}\right)-a_{t}^{+}\left(w^{\prime}\left(z_{t}\right)\right)+M\left(z ; z_{t}\right)\right) .
\end{aligned}
$$

We have further to satisfy the condition that the function (205) vanishes at all the $n-2$ zeros $d_{\nu}$ of $w^{\prime}(z)$. We may transform these conditions by using the formulas $\left(109^{\prime}\right)$ and $\left(110^{\prime}\right)$ to eliminate $N\left(z ; z_{t}\right)$ and $M\left(z ; z_{t}\right)$; we obtain

$$
\begin{array}{r}
\operatorname{Re}\left\{A-\frac{1}{i} \sum_{t=1}^{N} a_{t} w^{\prime}\left(z_{t}\right) \pi^{\prime}\left(z_{t} ; d_{\nu}\right)\right\}=0, \quad \nu=1,2, \cdots, n-2, \\
\operatorname{Im}\left\{A-\frac{1}{i} \sum_{t=1}^{N} a_{t} w^{\prime}\left(z_{t}\right)\left[p^{\prime}\left(z_{t} ; d_{\nu}\right)-\sum_{\mu=1}^{n} l_{\mu}\left(z_{\nu}\right) \omega_{\mu}\left(d_{\nu}\right)\right]\right\}=0, \\
\nu=1,2, \cdots, n-2 .
\end{array}
$$

From (204), (78) and (107') we conclude that

$$
\operatorname{Re}\left\{\sum_{t=1}^{N} a_{t} w^{\prime}\left(z_{t}\right) l_{\mu}\left(z_{t}\right)\right\}=0, \quad \mu=1,2, \cdots, n .
$$

Sending $z$ in (205) to the boundary $C$ of $D$ and using (57) and (206"), we find 
that $A$ is real. This leads to the conditions:

$$
\begin{array}{ll}
\operatorname{Im}\left\{\sum_{t=1}^{N} a_{t} w\left(z_{t}\right)\left[\pi^{\prime}\left(z_{t} ; d_{v}\right)-\pi^{\prime}\left(z_{t} ; d_{1}\right)\right]\right\}=0, & \nu=2,3, \cdots, n-2, \\
\operatorname{Re}\left\{\sum_{t=1}^{N} a_{t} w w^{\prime}\left(z_{t}\right) p^{\prime}\left(z_{t} ; d_{v}\right)\right\}=0, & \nu=1,2, \cdots, n-2 .
\end{array}
$$

We have in (204), (207) and (208) exactly our $3 n-6$ conditions (181), (183) and (184) for the parameters $a_{t}, z_{t}$ of our variation after passage to the limit $\rho \rightarrow 0$. It is interesting that the fullfillment of these conditions for a particular function $w^{\prime}(z)$ guarantees the equations for every function of this type.

\section{BIBLIOGRAPHY} 1941.

1. S. Bergman, Partial differential equations, Brown University Publications, Providence,

2. S. Bergman and M. Schiffer, A representation of Green's and Neumann'sfunctions in the theory of partial differential equations of second order, Duke Math. J. vol. 14 (1947) pp. 609-638.

3. B. Epstein, Some inequalities relating to conformal mapping upon canonical slit-domains, Bull. Amer. Math. Soc. vol. 53 (1947) pp. 813-819.

4. H. Groetzsch, Ueber die Verzerrung bei schlichter konformer Abbildung mehrfach zusammenhaengender schlichter Bereiche III, Berichte der Saechsische Akademie der Wissenschaften, Leipzig, vol. 83 (1931) pp. 283-297.

5. - Ueber das Parallelschlitztheorem der konformen Abbildung schlichter Bereiche, Berichte der Saechsischen Akademie der Wissenschaften, Leipzig, vol. 84 (1932) pp. 15-36.

6. H. Grunsky, Neue Abschaetzungen zur konformen Abbildung ein-und mehrfach zusammenhaengender Bereiche, Schriften des mathematischen Seminars und des Instituts für angewandte Mathematik an der Universität Berlin vol. 1 (1932) pp. 94-140.

7. - Koeffizientenbedingungen fuer schlicht abbildende meromorphe Funktionen, Math. Zeit. vol. 45 (1939) pp. 29-61.

8. K. Hensel and G. Landsberg, Theorie der algebraischen Functionen einer Variabeln und ihre Anwendung auf algebraische Kurven und algebraische Integrale, Leipzig, 1902.

9. M. Schiffer, $A$ method of variation within the family of simple functions, Proc. London Math. Soc. (2) vol. 44 (1938) pp. 432-449.

10. - Variation of the Green function and theory of the p-valued functions, Amer. J. Math. vol. 65 (1943) pp. 341-360. 216.

11. - The span of multiply connected domains, Duke Math. J. vol. 10 (1943) pp. 209-

12. - Hadamard's formula and variation of domain functions, Amer. J. Math. vol. 68 (1946) pp. 417-448.

13. - The kernel function of an orthonormal system, Duke Math. J. vol. 13 (1946) pp. 529-540.

14. - An application of orthonormal functions in the theory of conformal mapping, Amer. J. Math. vol. 70 (1948) pp. 147-156.

15. E. Schottky, Ueber die konforme Abbildung mehrfach zusammenhaengender ebener Flaechen, J. Reine Angew. Math. vol. 83 (1877) pp. 300-351.

HARVARD UNIVERSITY,

Cambridge, Mass. 